TRANSACTIONS OF THE

AMERICAN MATHEMATICAL SOCIETY

Volume 355, Number 12, Pages 5091-5117

S 0002-9947(03)03360-9

Article electronically published on July 24, 2003

\title{
CODIMENSION GROWTH AND MINIMAL SUPERALGEBRAS
}

\author{
A. GIAMBRUNO AND M. ZAICEV
}

\begin{abstract}
A celebrated theorem of Kemer (1978) states that any algebra satisfying a polynomial identity over a field of characteristic zero is PI-equivalent to the Grassmann envelope $G(A)$ of a finite dimensional superalgebra $A$. In this paper, by exploiting the basic properties of the exponent of a PI-algebra proved by Giambruno and Zaicev (1999), we define and classify the minimal superalgebras of a given exponent over a field of characteristic zero. In particular we prove that these algebras can be realized as block-triangular matrix algebras over the base field.

The importance of such algebras is readily proved: $A$ is a minimal superalgebra if and only if the ideal of identities of $G(A)$ is a product of verbally prime T-ideals. Also, such superalgebras allow us to classify all minimal varieties of a given exponent i.e., varieties $\mathcal{V}$ such that $\exp (\mathcal{V})=d \geq 2$ and $\exp (\mathcal{U})<d$ for all proper subvarieties $\mathcal{U}$ of $\mathcal{V}$. This proves in the positive a conjecture of Drensky (1988). As a corollary we obtain that there is only a finite number of minimal varieties for any given exponent. A classification of minimal varieties of finite basic rank was proved by the authors (2003).

As an application we give an effective way for computing the exponent of a T-ideal given by generators and we discuss the problem of what functions can appear as growth functions of varieties of algebras.
\end{abstract}

\section{INTRODUCTION}

Let $F$ be a field of characteristic zero and $F\langle X\rangle$ the free associative algebra of countable rank over $F$. Methods of representation theory of the symmetric group or of the general linear group have been a very useful tool in the study of the Tideals of $F\langle X\rangle$. Recall that the theory of T-ideals is strictly related to the theory of PI-algebras (or algebras satisfying a polynomial identity). It is well known and easy to see that every T-ideal $I$ of $F\langle X\rangle$ is the ideal of polynomial identities satisfied by some $F$-algebra $A$, and in this case we write $I=I d(A)$.

Regev in [26] proved that the tensor product of two PI-algebras is still PI. The proof was based on a comparative study of the asymptotic behaviour of a numerical sequence attached to every T-ideal. Such a sequence is called the sequence of codimensions of the T-ideal $I$ (or of a corresponding PI-algebra). His main result in this direction was proving that for any proper T-ideal such a sequence is exponentially bounded (see also [23]). More precisely, for every $n \geq 1$, one considers the space $P_{n}$

Received by the editors June 12, 2002 and, in revised form, March 20, 2003.

2000 Mathematics Subject Classification. Primary 16R10; Secondary 16P90.

Key words and phrases. Polynomial identity, T-ideal, superalgebra, variety, growth.

The first author was supported in part by MIUR of Italy.

The second author was partially supported by RFBR, grants 02-01-00219 and 00-15-96128. 
of multilinear polynomials of degree $n$ in a fixed set of variables and for a T-ideal $I$ defines $c_{n}(I)=\operatorname{dim}_{F} \frac{P_{n}}{P_{n} \cap I}$, the $n$th codimension of $I$.

In [11] and [12] the authors were able to determine the exponential behaviour of the sequence of codimensions $\left\{c_{n}(I)\right\}_{n \geq 1}$ for any proper T-ideal $I$. They proved that for any such T-ideal, $\exp (I)=\lim _{n \rightarrow \infty} \sqrt[n]{c_{n}(I)}$ always exists and is a non-negative integer. We call it the exponent of $I$. They also gave a constructive way for computing such an integer. Since every T-ideal $I$ uniquely determines a variety of associative algebras $\mathcal{V}=\mathcal{V}(I)$ we also write $\exp (I)=\exp (\mathcal{V})=\exp (A)$ for any algebra $A$ generating the variety $\mathcal{V}$ or $\operatorname{Id}(A)=I$.

Having at hand a scale provided by the exponent it becomes important to study T-ideals with the same exponent and to determine those with the most distinguished properties. To this end, in this paper we introduce the notion of a minimal superalgebra and we relate it to that of a verbally prime T-ideal and minimal variety.

Recall that the $\mathbb{Z}_{2}$-graded algebras or superalgebras and their identities are an important ingredient in the theory of PI-algebras developed by Kemer ([21]). If $G$ is the infinite dimensional Grassmann algebra over $F$ with its natural $\mathbb{Z}_{2}$-grading $G=G^{(0)} \oplus G^{(1)}$ and $A=A^{(0)} \oplus A^{(1)}$ is any superalgebra, then the Grassmann envelope of $A$ is $G(A)=\left(A^{(0)} \otimes G^{(0)}\right) \oplus\left(A^{(1)} \otimes G^{(1)}\right)$. A celebrated theorem of Kemer says that every T-ideal of $F\langle X\rangle$ is the ideal of identities $I=\operatorname{Id}(G(A))$ of the Grassmann envelope of a suitable finite dimensional superalgebra $A$. Another essential ingredient of the theory is the notion of a verbally prime T-ideal. Such ideals were classified, and it turns out that any proper T-ideal is the ideal of identities of an algebra of the type $G(A)$, where $A$ is an algebra of matrices with suitable grading.

The connection between the minimal superalgebras introduced in this paper and the above notions is the following. We prove that a proper T-ideal $I$ is a product of verbally prime T-ideals if and only if $I=I d(G(A))$ for some minimal superalgebra $A$. The exponent of the Grassmann envelope of a minimal superalgebra is readily computed: if $A=A_{s s}+J$ is the Wedderburn-Malcev decomposition of $A$ where $A_{s s}$ is a maximal semisimple $\mathbb{Z}_{2}$-stable subalgebra of $A$ and $J$ is the Jacobson radical, then $\exp (G(A))=\operatorname{dim} A_{\text {ss }}$.

The minimal superalgebras have a nice representation as upper block triangular matrices over the algebraically closed field $F$ with possibly various different $\mathbb{Z}_{2}$ gradings. In case $A$ is a superalgebra with trivial grading, such a representation is uniquely determined. In section 5 we give some examples.

Another important concept related to the minimal superalgebras is that of minimal variety of a given exponent. Recall that a variety $\mathcal{V}$ (or the corresponding T-ideal $I=I d(\mathcal{V}))$ is minimal of exponent $d \geq 2$ if $\exp (\mathcal{V})=d$ and $\exp (\mathcal{U})<d$ for all proper subvarieties $\mathcal{U}$ of $\mathcal{V}$.

Drensky in $[7$ and 8 proved that any variety whose T-ideal is generated by a product of commutators of length 2 or of length 3 is minimal. He also conjectured that a variety $\mathcal{V}$ is minimal if and only if its T-ideal of identities is a product of verbally prime T-ideals.

Another example of minimal variety was found in [30. Recently in 13], [14 the authors solved Drensky's conjecture in the positive for so-called varieties of finite basic rank. Recall that a variety $\mathcal{V}$ is of finite basic rank if $\mathcal{V}$ is generated by a finitely generated PI-algebra. In this case, as it was shown in [13], [14, $\mathcal{V}$ is a 
minimal variety if and only if $\mathcal{V}$ is generated by an upper block-triangular matrix algebra. Here we give a complete solution of Drensky's conjecture by showing that a variety $\mathcal{V}$ is minimal of exponent $d$ if and only if $\mathcal{V}$ is generated by the Grassmann envelope of a minimal superalgebra $A$. Hence the conjecture follows from the above-mentioned result. As a consequence of the classification of minimal varieties, it follows that surprisingly there are only a finite number of minimal varieties of given exponent. The main results of this paper were announced in [15.

In the last section of this paper we give two applications of the theory developed on minimal superalgebras. In the first we show how one can determine the exponent of a T-ideal given by an explicit set of generators. In the second application we discuss a conjecture of Regev stating that the codimension sequence of any PIalgebra $A$ behaves asymptotically as $C n^{e} d^{n}$ where $C$ is a constant, $e \in \frac{1}{2} \mathbb{Z}$ and $d \in \mathbb{Z}$. We get some results on the so-called inverse problem by exhibiting PIalgebras with prescribed growth.

The present paper came out as a natural continuation of the results in [14] (announced in [13]). Even though it is based on the development of the basic ideas of that paper, we must point out that some of the techniques and ideas in this paper are close to those of the paper [5] by Berele and Regev.

Throughout $F$ is a field of characteristic zero and $F\langle X\rangle=F\left\langle x_{1}, x_{2}, \ldots\right\rangle$ is the free associative algebra of countable rank over $F$. When needed, we shall also use different symbols for the variables of $X$.

Recall that a polynomial identity on an algebra $A$ is a polynomial

$$
f=f\left(x_{1}, \ldots, x_{n}\right) \in F\langle X\rangle
$$

vanishing under all valuations in $A$. If $f \neq 0$ we say that $f$ is non-trivial. The algebra $A$ is a PI-algebra if it satisfies a non-trivial polynomial identity. If $f$ is a polynomial identity on $A$ we usually write $f \equiv 0$ in $A$. Let $\operatorname{Id}(A)=\{f \in F\langle X\rangle \mid$ $f \equiv 0$ in $A\}$ be the T-ideal of identities of $A$. Recall that $\operatorname{Id}(A)$ is invariant under all endomorphisms of $F\langle X\rangle$. If $\mathcal{V}$ is a variety of associative algebras, $\mathcal{V}$ uniquely determines a T-ideal $I$ and we write $I=I d(\mathcal{V})$. Also, if $\mathcal{V}$ is generated by the algebra $A$ we write $\mathcal{V}=\operatorname{var}(A)$. We refer the reader to the books [29] and [9] for an account of the basic properties of PI-algebras.

Since $\operatorname{char} F=0$, by the well-known multilinearization process, every T-ideal is determined by its multilinear polynomials, and we shall tacitly use this fact throughout the paper.

\section{Supercommutative envelopes}

In this section we shall define the superenvelope or $S$-envelope of a superalgebra and we shall compare it to the Grassmann envelope of the algebra.

Recall that an $F$-algebra $A$ is a $\mathbb{Z}_{2}$-graded algebra (or superalgebra) if $A$ has a vector space decomposition $A=A^{(0)} \oplus A^{(1)}$ such that $A^{(0)} A^{(0)}+A^{(1)} A^{(1)} \subseteq A^{(0)}$ and $A^{(0)} A^{(1)}+A^{(1)} A^{(0)} \subseteq A^{(1)}$. The elements of $A^{(0)}$ are called homogeneous of degree 0 (or of even degree) and those of $A^{(1)}$ are called homogeneous of degree 1 (or of odd degree).

A superalgebra $A=A^{(0)} \oplus A^{(1)}$ is said to be supercommutative if for all homogeneous elements $a, b \in A$ we have that

$$
a b-(-1)^{(\operatorname{deg} a)(\operatorname{deg} b)} b a=0 .
$$


One defines in an obvious way a free object $S$ of countable rank in the variety of supercommutative superalgebras as follows. Take $U=\left\{u_{1}, u_{2}, \ldots\right\}$ and $V=\left\{v_{1}, v_{2}, \ldots\right\}$ be two countable sets and define $S=F[U, V]$ to be the algebra generated by $U \cup V$ over $F$ subject to the conditions that the elements of $U$ are central and the elements of $V$ anticommute. In symbols,

$$
S=\left\langle u_{1}, v_{1}, u_{2}, v_{2}, \ldots \mid u_{i} u_{j}=u_{j} u_{i}, v_{i} v_{j}=-v_{j} v_{i}, u_{i} v_{j}=v_{j} u_{i}, i, j=1,2, \ldots\right\rangle .
$$

$S=F[U, V]$ is called the free supercommutative algebra over $F$ on the countable sets of commuting variables $U$ and anticommuting variables $V$; it has a natural $\mathbb{Z}_{2}$-grading $S=S^{(0)} \oplus S^{(1)}$ if we require the variables of $U$ to be even and those of $V$ to be odd. The algebra $S$ has the following universal property: given any supercommutative algebra $A$, any map $\varphi:\{U, V\} \rightarrow A$ such that $\varphi(U) \subseteq A^{(0)}$ and $\varphi(V) \subseteq A^{(1)}$ can be uniquely extended to a homomorphism of superalgebras $\bar{\varphi}: S \rightarrow A$.

A useful example of supercommutative algebra is given by $G$, the Grassmann algebra on a countable dimensional vector space. We may clearly assume that

$$
G=\left\langle v_{1}, v_{2}, \ldots \mid v_{i} v_{j}=-v_{j} v_{i}, i, j=1,2, \ldots\right\rangle
$$

so that $G \subseteq S$ and $G=G^{(0)} \oplus G^{(1)}$ is the induced $\mathbb{Z}_{2}$-grading. Thus $G^{(0)}$ is spanned by all monomials in the $v_{i}$ 's of even length and $G^{(1)}$ is spanned by all monomials in the $v_{i}$ 's of odd length.

Given any superalgebra $A=A^{(0)} \oplus A^{(1)}$, the Grassmann envelope of $A$ is the superalgebra $G(A)=\left(A^{(0)} \otimes G^{(0)}\right) \oplus\left(A^{(1)} \otimes G^{(1)}\right)$. This has been a very useful tool in PI-theory: Kemer showed that any variety of algebras can be generated by the Grassmann envelope of a suitable finite dimensional superalgebra ([21]). As for the Grassmann algebra, one can define the so-called superenvelope or $S$-envelope of any superalgebra.

Definition 2.1. If $A=A^{(0)} \oplus A^{(1)}$ is a superalgebra, the $S$-envelope of $A$ is the superalgebra

$$
S(A)=\left(A^{(0)} \otimes S^{(0)}\right) \oplus\left(A^{(1)} \otimes S^{(1)}\right) .
$$

It is not difficult to see that actually $G(A)$ and $S(A)$ have the same identities. We prove this fact in the next lemma.

Lemma 2.2. Let $A=A^{(0)} \oplus A^{(1)}$ be a superalgebra. Then

1) $S(A)$ and $G(A)$ satisfy the same polynomial identities;

2) if $\operatorname{dim}_{F} A<\infty$ and $\left\{a_{1}, \ldots, a_{k}\right\},\left\{b_{1}, \ldots, b_{t}\right\}$ are bases of $A^{(0)}$ and $A^{(1)}$ respectively, then the subalgebra of $S(A)$ generated by the elements

$\xi_{i}=a_{1} \otimes u_{i 1}+\cdots+a_{k} \otimes u_{i k}+b_{1} \otimes v_{i 1}+\cdots+b_{t} \otimes v_{i t}, i=1,2, \ldots$,

is a relatively free algebra of the variety $\operatorname{var}(G(A))$ with free generators $\xi_{1}, \xi_{2}, \ldots$

Proof. As we remarked above, $G \subseteq S$ is the subalgebra generated by $V$. Hence $S(A) \supseteq G(A)$ and $G(A)$ satisfies all the identities of $S(A)$.

Now let $f\left(x_{1}, \ldots, x_{n}\right)$ be a multilinear polynomial which is not an identity of $S(A)$. Then there exist $a_{1}, \ldots, a_{n} \in A^{(0)} \cup A^{(1)}$ and $p_{1}, \ldots, p_{n} \in S^{(0)} \cup S^{(1)}$ such that

$$
f\left(a_{1} \otimes p_{1}, \ldots, a_{n} \otimes p_{n}\right) \neq 0 .
$$


We may clearly assume that $a_{1}, \ldots, a_{r} \in A^{(0)}, p_{1}, \ldots, p_{r} \in S^{(0)}, a_{r+1}, \ldots, a_{n} \in$ $A^{(1)}$, and $p_{r+1}, \ldots, p_{n} \in S^{(1)}$. Hence, recalling that the $p_{i}$ 's commute or anticommute among themselves, we can write

$$
f\left(a_{1} \otimes p_{1}, \ldots, a_{n} \otimes p_{n}\right)=b \otimes p_{1} \cdots p_{n}
$$

with $0 \neq b \in A$ and $0 \neq p_{1} \cdots p_{n} \in S$. It is clear that the same computations show that

$$
f\left(a_{1} \otimes u_{1}, \ldots, a_{r} \otimes u_{r}, a_{r+1} \otimes v_{1}, \ldots, a_{n} \otimes v_{n-r}\right)=b \otimes u_{1} \cdots u_{r} v_{1} \cdots v_{n-r} \neq 0 .
$$

Since $S$ is the free supercommutative algebra, the map $\varphi: A \otimes\{U, V\} \rightarrow A \otimes G$ such that

$$
\varphi\left(a \otimes v_{i}\right)=a \otimes v_{i}, \varphi\left(a \otimes u_{i}\right)=a \otimes v_{n+2 i-1} v_{n+2 i}, i=1,2, \ldots,
$$

for all $a \in A$, can be extended to a homomorphism $\bar{\varphi}: A \otimes S \rightarrow A \otimes G$ such that $\bar{\varphi}(S(A)) \subseteq G(A)$. We obtain

$$
\begin{gathered}
\bar{\varphi}\left(f\left(a_{1} \otimes u_{1}, \ldots, a_{r} \otimes u_{r}, a_{r+1} \otimes v_{1}, \ldots, a_{n} \otimes v_{n-r}\right)\right) \\
=f\left(a_{1} \otimes v_{n+1} v_{n+2}, \ldots, a_{r} \otimes v_{n+2 r-1} v_{n+2 r}, a_{r+1} \otimes v_{1}, \ldots, a_{n} \otimes v_{n-r}\right) \\
=b \otimes v_{n+1} v_{n+2} \cdots v_{n+2 r-1} v_{n+2 r} v_{1} \cdots v_{n-r} \neq 0 .
\end{gathered}
$$

Hence $f$ is not an identity of $G(A)$, and the first part of the lemma is proved.

Suppose now that $A$ is a finite dimensional superalgebra and let

$$
A^{(0)}=\operatorname{Span}_{F}\left\{a_{1}, \ldots, a_{k}\right\}, A^{(1)}=\operatorname{Span}_{F}\left\{b_{1}, \ldots, b_{t}\right\} .
$$

Let $f\left(x_{1}, \ldots, x_{n}\right)$ be a non-zero polynomial and suppose that $f\left(\xi_{1}, \ldots, \xi_{n}\right)=0$. If $c_{1}, \ldots, c_{n} \in S(A)$, then, for all $1 \leq i \leq n$, we can write

$$
c_{i}=a_{1} \otimes p_{i 1}+\cdots+a_{k} \otimes p_{i k}+b_{1} \otimes q_{i 1}+\cdots+b_{t} \otimes q_{i t}
$$

for suitable $p_{i j} \in S^{(0)}, q_{i j} \in S^{(1)}$. As above, there exists a homomorphism $\bar{\varphi}$ : $A \otimes S \rightarrow A \otimes S$ such that $\bar{\varphi}\left(a_{i}\right)=a_{i}, \bar{\varphi}\left(b_{j}\right)=b_{j}, \bar{\varphi}\left(u_{i j}\right)=p_{i j}, \bar{\varphi}\left(v_{i j}\right)=q_{i j}, 1 \leq i \leq$ $k, 1 \leq j \leq t$. It follows that $f\left(c_{1}, \ldots, c_{n}\right)=\bar{\varphi}\left(f\left(\xi_{1}, \ldots, \xi_{n}\right)\right)=0$ and $f$ is an identity for the algebra $S(A)$. This says that the algebra generated by the elements $\xi_{1}, \xi_{2}, \ldots$ is a relatively free algebra of the variety $\operatorname{var}(S(A))$. Since $\operatorname{var}(S(A))=\operatorname{var}(G(A))$, the proof is complete.

\section{Minimal Superalgebras}

Throughout this section we shall assume that $F$ is an algebraically closed field of characteristic zero. It is well known (see 21]) that any finite dimensional simple superalgebra over $F$ is of one of the following three types:

1) $M_{n}(F)$, the algebra of $n \times n$ matrices over $F$ with trivial grading $\left(M_{n}(F), 0\right)$.

2) $M_{k, l}(F)=\left(\begin{array}{cc}A & B \\ C & D\end{array}\right)$ where $A \in M_{k}(F), D \in M_{l}(F), B$ is a $k \times l$ matrix and $C$ is an $l \times k$ matrix, with grading $\left(\left(\begin{array}{cc}A & 0 \\ 0 & D\end{array}\right),\left(\begin{array}{cc}0 & B \\ C & 0\end{array}\right)\right), 1 \leq k \leq \frac{n}{2}$.

3) $M_{n}(F \oplus t F)$, where $t^{2}=1$ with grading $\left(M_{n}(F), t M_{n}(F)\right)$.

The following lemma holds.

Lemma 3.1. Let $A$ be a simple superalgebra of type 1), 2) or 3). Then there exist orthogonal idempotents $e_{1}, \ldots, e_{n} \in A^{(0)}$ such that $e_{1}+\cdots+e_{n}=1$ and for every $i=1, \ldots, n, A e_{i}\left(e_{i} A\right)$ is a minimal left (resp. right) graded ideal of $A$. 
The proof of the above lemma is obvious. It is enough to take $e_{1}, \ldots, e_{n}$ to be all diagonal matrix units in case 1 ) or 2 ) or all diagonal matrix units of $A^{(0)}$ in case 3).

Definition 3.2. The idempotents of Lemma 3.1 are called the minimal graded idempotents of the simple superalgebra $A$.

Note that they are all homogeneous and lie in $A^{(0)}$.

Now let $A=A^{(0)} \oplus A^{(1)}$ be a finite dimensional superalgebra. For arbitrary $a=a_{0}+a_{1}$ with $a_{0} \in A^{(0)}, a_{1} \in A^{(1)}$ we define $\varphi(a)=a_{0}-a_{1}$. Then $\varphi$ is an automorphism of $A$ of order 2 and a subspace $V \subseteq A$ is homogeneous in the $\mathbb{Z}_{2}$-grading if and only if $\varphi(V) \subseteq V$. It follows that $J=J(A)$, the Jacobson radical of $A$, is homogeneous in the $\mathbb{Z}_{2}$-grading $J=J^{(0)} \oplus J^{(1)}$. Moreover by the generalization of the Wedderburn-Malcev theorem given in 31] we can write $A=A_{s s}+J$, where $A_{s s}$ is a maximal semisimple subalgebra homogeneous in the $\mathbb{Z}_{2^{-}}$ grading. Also $A_{s s}$ can be written as the direct sum of graded simple superalgebras and since $F$ is algebraically closed, $A_{s s}=A_{1} \oplus \cdots \oplus A_{m}$, where $A_{1}, \ldots, A_{m}$ are simple superalgebra of type 1), 2) or 3) above.

Definition 3.3. A superalgebra $A$ is minimal if it is finite dimensional and $A=$ $A_{s s}+J$ where

1) $A_{s s}=A_{1} \oplus \cdots \oplus A_{m}$ with $A_{1}, \ldots, A_{m}$ simple superalgebras of type 1$), 2$ ) or 3$)$;

2 ) there exist homogeneous elements $w_{12}, \ldots, w_{m-1, m} \in J^{(0)} \cup J^{(1)}$ and minimal graded idempotents $e_{1} \in A_{1}, \ldots, e_{m} \in A_{m}$ such that

$$
e_{i} w_{i, i+1}=w_{i, i+1} e_{i+1}=w_{i, i+1}, \quad i=1, \ldots, m-1,
$$

and

$$
w_{12} w_{23} \cdots w_{m-1, m} \neq 0
$$

3) $w_{12}, \ldots, w_{m-1, m}$ generate $J$ as a two-sided ideal of $A$.

Lemma 3.4. Let $\mathcal{V}$ be a variety of algebras over the algebraically closed field $F$. If $\exp (\mathcal{V}) \geq 2$, then there exists a minimal superalgebra $A$ with maximal semisimple subalgebra $A_{\text {ss }}$ such that $G(A) \in \mathcal{V}$ and $\exp (\mathcal{V})=\operatorname{dim}_{F} A_{\text {ss }}$.

Proof. By a theorem of Kemer ([21]) there exists a finite dimensional superalgebra $B$ such that $\mathcal{V}=\operatorname{var}(G(B))$. Let $B=B_{s s}+J$ where $B_{s s}=A_{1} \oplus \cdots \oplus A_{n}$ with $A_{1}, \ldots, A_{n}$ simple superalgebras. Then, by the characterization of the exponent given in [12] it follows that there exists $1 \leq m \leq n$ such that $A_{1} J A_{2} J \cdots J A_{m} \neq$ 0 and $\exp (\mathcal{V})=\operatorname{dim}\left(A_{1} \oplus \cdots \oplus A_{m}\right)$. Hence for some $x_{1}, \ldots, x_{m-1} \in J, a_{1} \in$ $A_{1}, \ldots, a_{m} \in A_{m}$ we have that

$$
a_{1} x_{1} a_{2} \cdots a_{m-1} x_{m-1} a_{m} \neq 0 .
$$

Moreover we may clearly assume that the elements $x_{1}, \ldots, x_{m-1}, a_{1}, \ldots, a_{m}$ are homogeneous in the $\mathbb{Z}_{2}$-grading. Let $1_{1}, \ldots, 1_{m}$ be the unit elements of the algebras $A_{1}, \ldots, A_{m}$, respectively. Then from the above inequality we can write

$$
1_{1}\left(a_{1} x_{1} a_{2}\right) 1_{2}\left(x_{2} a_{3}\right) 1_{3} \cdots 1_{m-1}\left(x_{m-1} a_{m}\right) 1_{m} \neq 0 .
$$

If we now decompose all unit elements $1_{1}, \ldots, 1_{m}$ into minimal graded idempotents (cf. Lemma 3.1), we obtain that for some $e_{1} \in A_{1}, \ldots, e_{m} \in A_{m}$,

$$
e_{1} y_{1} e_{2} y_{2} \cdots y_{m-1} e_{m} \neq 0
$$


where $y_{1}=a_{1} x_{1} a_{2}, y_{2}=x_{2} a_{3}, \ldots, y_{m-1}=x_{m-1} a_{m}$. Finally, define

$$
w_{12}=e_{1} y_{1} e_{2}, w_{23}=e_{2} y_{2} e_{3}, \ldots, w_{m-1, m}=e_{m-1} y_{m-1} e_{m} .
$$

Clearly the elements $w_{12}, \ldots, w_{m-1, m}$ are homogeneous in the $\mathbb{Z}_{2}$-grading and belong to $J$. Also $e_{i} w_{i, i+1}=w_{i, i+1} e_{i+1}=w_{i, i+1}$, for all $i=1, \ldots, m-1$. Now let $A$ be the algebra generated by $A_{1}, \ldots, A_{m}$ and $w_{12}, \ldots, w_{m-1, m}$. Then $A_{s s}=A_{1} \oplus \cdots \oplus A_{m}$ is such that $\operatorname{dim} A_{s s}=\exp (\mathcal{V})$ and $G(A) \subseteq G(B)$ implies that $G(A) \in \mathcal{V}$.

We remark that the algebra $A$ constructed in the previous lemma is not uniquely determined. At the end of section 5 we shall give few examples in order to show that even if two minimal superalgebras have the same semisimple part still they might be of different dimension over the base field $F$. Nevertheless their Grassmann envelopes satisfy the same identities.

In the next lemmas we describe the structure of a generic minimal superalgebra. We fix the notation for the next two lemmas: $A$ will be a minimal superalgebra, such that $A=A_{s s}+J$ with $A_{s s}=A_{1} \oplus \cdots \oplus A_{m}, A_{1}, \ldots, A_{m}$ simple superalgebras, and $w_{12}, \ldots, w_{m-1, m} \in J$ are the homogeneous elements satisfying conditions 2 ) and 3) of the definition.

Lemma 3.5. The minimal superalgebra $A$ has the following vector space decomposition:

$$
A=\bigoplus_{1 \leq i \leq j \leq m} A_{i j}
$$

where $A_{11}=A_{1}, \ldots, A_{m m}=A_{m}$ and, for all $i<j$,

$$
A_{i j}=A_{i} w_{i, i+1} A_{i+1} \cdots A_{j-1} w_{j-1, j} A_{j} .
$$

Moreover $J=\bigoplus_{i<j} A_{i j}$ and $A_{i j} A_{k l}=\delta_{j k} A_{i l}$, where $\delta_{j k}$ is the Kronecker delta.

Proof. From property 2) of the definition of minimal superalgebra, a product of the type

$$
a_{1} w_{i_{1}, i_{1}+1} a_{2} \cdots a_{k} w_{i_{k}, i_{k}+1} a_{k+1}
$$

with $a_{1}, \ldots, a_{k} \in A_{s s}$ is non-zero only if $i_{1}+1=i_{2}, \ldots, i_{k-1}+1=i_{k}$ and $a_{1} \in$ $A_{i_{1}}, \ldots, a_{k+1} \in A_{i_{k}+1}$ (hence the product belongs to $\left.A_{i_{1}, i_{k+1}}\right)$. It follows that $A$ is the sum of the subspaces $A_{i j}$ with $1 \leq i \leq j \leq m$. It is also clear that $J=\sum_{i<j} A_{i j}$. The orthogonality of the graded idempotents $e_{1} \in A_{1}, \ldots, e_{m} \in A_{m}$ guarantees that the sum is direct and $A_{i j} A_{k l}=\delta_{j k} A_{i l}$.

We now assume that $m>2$ and we investigate the structure of the spaces $w_{i-1, i} A_{i} w_{i, i+1}$, for $i=2, \ldots, m-1$.

If $A_{i}$ is a simple superalgebra of type 1) or 2), then $e_{i} A_{i} e_{i}=\operatorname{Span}\left\{e_{i}\right\}$ and

$$
w_{i-1, i} A_{i} w_{i, i+1}=w_{i-1, i} e_{i} A_{i} e_{i} w_{i, i+1}=\operatorname{Span}\left\{w_{i-1, i} w_{i, i+1}\right\} .
$$

If $A_{i}=M_{n_{i}}\left(F \oplus t_{i} F\right)$ with $t_{i}^{2}=1$ is of type 3$)$, then $e_{i} A_{i} e_{i}$ is spanned by the elements $e_{i}$ and $t_{i} e_{i}$. Hence

$$
w_{i-1, i} A_{i} w_{i, i+1}=\operatorname{Span}\left\{w_{i-1, i} w_{i, i+1}, w_{i-1, i} t_{i} w_{i, i+1}\right\}
$$

and, in general, the elements $w_{i-1, i} w_{i, i+1}$ and $w_{i-1, i} t_{i} w_{i, i+1}$ are not linearly independent. Note that from the inequality $w_{12} w_{23} \cdots w_{m-1, m} \neq 0$ it follows that $w_{i-1, i} w_{i, i+1} \neq 0$. 
By extending this procedure for any $i, j$ with $j-i \geq 2$, we define

$$
w_{i j}\left(q_{i+1}, \ldots, q_{j-1}\right)=w_{i, i+1} q_{i+1} w_{i+1, i+2} \cdots w_{j-2, j-1} q_{j-1} w_{j-1, j},
$$

where $q_{k}=1$ if $A_{k}$ is a simple superalgebra of type 1) or 2), and $q_{k}=1$ or $q_{k}=t_{k}$ if $A_{k}=M_{n_{k}}\left(F \oplus t_{k} F\right)$. For short we shall also write $w_{i j}$ for $w_{i j}(1, \ldots, 1)$.

The next lemma gives us the structure of $A_{i j}$ as an $\left(A_{i}, A_{j}\right)$-bimodule.

Lemma 3.6. Let $A$ be a minimal superalgebra. Then the elements

$$
w_{i j}\left(q_{i+1}, \ldots, q_{j-1}\right),
$$

for all possible values of $q_{i+1}, \ldots, q_{j-1}$, generate $A_{i j}$ as an $\left(A_{i}, A_{j}\right)$-bimodule. Moreover $w_{i j}=w_{i j}(1, \ldots, 1)$ generates a non-zero irreducible graded $\left(A_{i}, A_{j}\right)$-bimodule.

Proof. The first statement of the lemma is clear since

$$
A_{i j}=A_{i} w_{i, i+1} A_{i+1} \cdots A_{j-1} w_{j-1, j} A_{j}
$$

and $w_{i, i+1} A_{i+1} \cdots A_{j-1} w_{j-1, j}$ is spanned by the elements $w_{i j}\left(q_{i+1}, \ldots, q_{j-1}\right)$, for all possible values of $q_{i+1}, \ldots, q_{j-1}$. Now, for any $w=w_{i j}\left(q_{i+1}, \ldots, q_{j-1}\right) \neq 0$, we have that $e_{i} w=w e_{j}=w$. Hence $A_{i} w A_{j}$ is a non-zero irreducible graded $\left(A_{i}, A_{j}\right)$-bimodule.

\section{Grassmann envelopes of minimal superalgebras AND THEIR IDENTITIES}

In this section we keep the notation of the previous section. Hence $F$ will be an algebraically closed field unless otherwise stated. If $A$ is a minimal superalgebra we assume that $A$ has the decomposition $A=A_{s s}+J=A_{1} \oplus \cdots \oplus A_{m}+J$ and $w_{12}, \ldots, w_{m-1, m} \in J$ are the elements satisfying conditions 2$\left.), 3\right)$ of the definition. Now let $f=f\left(x_{1}, \ldots, x_{n}\right)$ be a multilinear polynomial in $F\langle X\rangle$ and let $B$ be an $F$ algebra. Let $f(B)$ denote the subspace of $B$ generated by all valuations $f\left(b_{1}, \ldots, b_{n}\right)$ with $b_{1}, \ldots, b_{n} \in B$. If $[$,$] denotes the Lie commutator [x, y]=x y-y x$, then, since $\left[f\left(b_{1}, \ldots, b_{n}\right), y\right]=f\left(\left[b_{1}, y\right], \ldots, b_{n}\right)+\cdots+f\left(b_{1}, \ldots,\left[b_{n}, y\right]\right)$, it follows that $f(B)$ is a Lie ideal of $B$.

Lemma 4.1. Let $A=A_{1} \oplus \cdots \oplus A_{m}+J$ be a minimal superalgebra and suppose that $f=f\left(x_{1}, \ldots, x_{n}\right)$ is a multilinear polynomial which is not an identity of $S(A)=$ $\left(A^{(0)} \otimes S^{(0)}\right) \oplus\left(A^{(1)} \otimes S^{(1)}\right)$, the $S$-envelope of $A$.

1) If $m \geq 2$, then $f(S(A))$ contains the element $w_{1 m} \otimes p$, for some non-zero monomial $p \in F[U, V]$.

2) If $m=1$, then either $E \otimes p \in f(S(A))$ for some non-zero monomial $p \in$ $F[U, V]$, where $E$ is the unit element of $A$ or, for any two minimal graded idempotents $e_{1}, e_{2} \in A$, there exists a non-zero monomial $p \in F[U, V]$ such that $\left(e_{1}-e_{2}\right) \otimes p \in f(S(A))$.

Proof. Suppose first that $m=1$, i.e., $A$ is a simple superalgebra. Since $f$ is not an identity of $S(A)$, there exist homogeneous elements $a_{1}, \ldots, a_{n} \in A$ and monomials $p_{1}, \ldots, p_{n} \in F[U, V]$ such that $f\left(a_{1} \otimes p_{1}, \ldots, a_{n} \otimes p_{n}\right)=a \otimes q \neq 0$ for some homogeneous elements $a \in A, q \in F[U, V]$.

We first claim that if $a$ is a central element of $A$, then the conclusion of the lemma follows. In fact, if $A$ is a simple superalgebra of type 1) or 2) (see section 3), then $a=\lambda E$ for some $\lambda \in F$ and we are done. If $A$ is of type 3), then, since $a$ is homogeneous, either $a=\lambda E$ or $a=\lambda t E$. In this last case one of the $a_{i}$ 's, say $a_{1}$, 
must be of the type $a_{1}=t a_{1}^{\prime}$ with $a_{1}^{\prime} \in A^{(0)}$. But then $f\left(a_{1}^{\prime} \otimes p_{1}, \ldots, a_{n} \otimes p_{n}\right)=$ $\lambda E \otimes p^{\prime}$ and we are also done in this case.

Therefore we may assume that $a$ is not central in $A$. Since $f(S(A))$ is a Lie ideal of $S(A)$, it contains $U \otimes p^{\prime}$, where $U$ is the Lie ideal of $A$ generated by $a$. If $A$ is a simple superalgebra of type 1 ) or 2), i.e., $A=M_{k}(F)$ with suitable grading, the only non-central Lie ideal of $A$ is $s l_{k}(F)$, the Lie algebra of $k \times k$ traceless matrices. Since in this case all minimal graded idempotents of $A$ are diagonal matrix units, it follows that $e_{1}-e_{2} \in U=\operatorname{sl}_{k}(F)$ and $\left(e_{1}-e_{2}\right) \otimes p^{\prime} \in f(S(A))$. If $A$ is simple of type 3 ), as above we may assume $a$ to be a non-central element of $A^{(0)}$. By applying the same arguments as when $A$ is simple of type 1 ) or 2), we complete the proof for $m=1$.

Suppose now that $m \geq 2$ and we proceed by induction on $m$. By the first part of the proof the case $m=1$ is settled. Suppose first that $f$ is not an identity of one of the simple summands, say $A_{i}, 1 \leq i \leq m$. By the definition of minimal superalgebra, there exists a minimal idempotent $e_{i} \in A_{i}$ such that $e_{i} w_{i, i+1}=w_{i, i+1}$, in the case $i<m$ and $w_{m-1, m} e_{m}=w_{m-1, m}$ in the case $i=m$. By the first part of the proof there exists $a \otimes p \in f\left(S\left(A_{i}\right)\right)$ such that either $a=E$, the unit element of $A_{i}$, or $a=e_{i}-e_{i}^{\prime}$ for some idempotent $e_{i}^{\prime} \in A_{i}$ orthogonal to $e_{i}$. In any case for $i<m$ we have

$$
\begin{gathered}
a w_{i, i+1} \cdots w_{m-1, m}=w_{i, i+1} \cdots w_{m-1, m}, \\
w_{i, i+1} \cdots w_{m-1, m} a=0
\end{gathered}
$$

and, for $i=m$, we have

$$
\begin{gathered}
w_{1 m} a=w_{12} \cdots w_{m-1, m} a=w_{12} \cdots w_{m-1, m}=w_{1 m}, \\
a w_{1 m}=a w_{12} \cdots w_{m-1, m}=0 .
\end{gathered}
$$

Let $p^{\prime}, p^{\prime \prime} \in S$ be such that $p^{\prime} p p^{\prime \prime} \neq 0$. Then, since $f\left(S\left(A_{i}\right)\right) \subseteq f(S(A))$ and $f(S(A))$ is a Lie ideal of $S(A)$, it follows that

$$
w_{1 m} \otimes p^{\prime} p p^{\prime \prime}=\left[w_{12} \cdots w_{i-1, i} \otimes p^{\prime},\left[a \otimes p, w_{i, i+1} \cdots w_{m-1, m} \otimes p^{\prime \prime}\right]\right] \in f(S(A))
$$

in the case $i<m$, and $w_{1 m} \otimes p^{\prime} p=\left[a \otimes p, w_{1 m} \otimes p^{\prime}\right] \in f(S(A))$ in the case $i=m$, proving the lemma.

Therefore we may assume that $f$ is an identity for $A_{s s}=A_{1} \oplus \cdots \oplus A_{m}$. Consider the following two subalgebras of $A$ :

$$
B=\bigoplus_{1 \leq i \leq j \leq m-1} A_{i j}, \quad C=\bigoplus_{2 \leq i \leq j \leq m} A_{i j} .
$$

Now, if $f$ is not an identity of $S(B)$, then by induction either $w_{1, m-1} \otimes p \in f(S(B))$ for some $p \neq 0$ or $E_{B} \otimes p \in f(S(B))$, where $E_{B}$ is the unit element of $B$ or $\left(e_{1}-e_{2}\right) \otimes p \in f(S(B))$ for any two minimal graded idempotents of $B$. Thus in the first case, if $p^{\prime} \in S$ is such that $p p^{\prime} \neq 0$, we get

$$
w_{1 m} \otimes p p^{\prime}=\left[w_{1, m-1} \otimes p, w_{m-1, m} \otimes p^{\prime}\right] \in f(S(A))
$$

and we are done. In the second case, $a w_{12}=w_{12}$ and $w_{12} a=0$ for $a=E_{B}$ or $a=e_{1}-e_{2}$. This implies that

$$
w_{1 m} \otimes p p^{\prime} p^{\prime \prime}=\left[\left[a \otimes p, w_{12} \otimes p^{\prime}\right], w_{23} \cdots w_{m-1, m} \otimes p^{\prime \prime}\right] \in f(S(A))
$$

and we are also done if we take $p^{\prime}, p^{\prime \prime} \in S$ such that $p p^{\prime} p^{\prime \prime} \neq 0$. Similarly if $f$ is not an identity for $S(C)$, we also get $w_{1 m} \otimes \bar{p} \in f(S(A))$ for some $\bar{p} \in S$. 
Therefore we may assume that $f$ is an identity of $S(B)$ and $S(C)$. In this case all non-zero values of $f$ belong to $S\left(A_{1 m}\right)$. Thus there exist homogeneous elements $u_{1}, \ldots, u_{n} \in A, p_{1}, \ldots, p_{n} \in S$ such that

$$
f\left(u_{1} \otimes p_{1}, \ldots, u_{n} \otimes p_{n}\right)=u \otimes p \neq 0
$$

and by Lemma 3.6 we have that $u=a w_{1 m}\left(q_{1}, \ldots, q_{m}\right) b$ for some $q_{1}, \ldots, q_{m}$ and for some $a \in A_{1}, b \in A_{m}$. Moreover some of the elements $u_{1}, \ldots, u_{n}$, say $u_{1}, \ldots, u_{k}$, belong to $J=\bigoplus_{i<j} A_{i j}$ and $u_{k+1}, \ldots, u_{n} \in A_{s s}$. Let

$$
\begin{gathered}
u_{1}=a_{i_{1}} w_{i_{1} j_{1}}\left(q_{i_{1}+1}^{(1)}, \ldots, q_{j_{1}-1}^{(1)}\right) b_{j_{1}} \\
\vdots \\
u_{k}=a_{i_{k}} w_{i_{k} j_{k}}\left(q_{i_{k}+1}^{(k)}, \ldots, q_{j_{k}-1}^{(k)}\right) b_{j_{k}}
\end{gathered}
$$

where $a_{i_{1}} \in A_{i_{1}}, b_{j_{1}} \in A_{j_{1}}, \ldots, a_{i_{k}} \in A_{i_{k}}, b_{j_{k}} \in A_{j_{k}}$.

Now replace in (4.1) the element $u_{1}$ with $\bar{u}_{1}=a_{i_{1}} w_{i_{1} j_{1}}(1, \ldots, 1) b_{j_{1}}, \ldots$ and the element $u_{k}$ with $\bar{u}_{k}=a_{i_{k}} w_{i_{k} j_{k}}(1, \ldots, 1) b_{j_{k}}$. Then, by the multiplication rules of $A$ we obtain

$$
f\left(\bar{u}_{1} \otimes p_{1}, \ldots, \bar{u}_{k} \otimes p_{k}, u_{k+1} \otimes p_{k+1}, \ldots, u_{n} \otimes p_{n}\right)=\bar{u} \otimes p
$$

where $\bar{u}=a w_{1 m}(1, \ldots, 1) b=a w_{1 m} b \neq 0$.

Recall that by the choice of $w_{i j}$ there exist minimal graded idempotents $e_{1} \in$ $A_{1}, e_{m} \in A_{m}$ such that $e_{1} w_{1 m}=w_{1 m} e_{m}=w_{1 m}$. Hence since $e_{1} \in A_{1} a e_{1}$ and $e_{m} \in e_{m} b A_{m}$, by taking $p^{\prime}, p^{\prime \prime} \in S$ such that $p^{\prime} p p^{\prime \prime} \neq 0$, we get

$$
\begin{aligned}
& 0 \neq w_{1 m} \otimes p^{\prime} p p^{\prime \prime} \in A_{1} a e_{1} w_{1 m} e_{m} b A_{m} \otimes p^{\prime} p p^{\prime \prime} \\
& =\left[\left[A_{1} \otimes p^{\prime}, a w_{1 m} b \otimes p\right], A_{m} \otimes p^{\prime \prime}\right] \subseteq f(S(A)) .
\end{aligned}
$$

This completes the proof of the lemma.

Lemma 4.2. If $A=A_{1} \oplus \cdots \oplus A_{m}+J$ is a minimal superalgebra, then $\operatorname{Id}\left(G\left(A_{1}\right)\right)$ $\cdots \operatorname{Id}\left(G\left(A_{m}\right)\right) \subseteq \operatorname{Id}(G(A))$.

Proof. Let $f_{1} \in \operatorname{Id}\left(G\left(A_{1}\right)\right), \ldots, f_{m} \in \operatorname{Id}\left(G\left(A_{m}\right)\right)$ and $f=f_{1} \cdots f_{m}$. If $m=1$, the conclusion of the lemma is clear. Suppose $m>1$ and let $A^{\prime}$ be the subalgebra of $A$ generated by $A_{1}, \ldots, A_{m-1}$ and $w_{12}, \ldots, w_{m-2, m-1}$. Then, by the inductive hypotheses, $f_{1} \cdots f_{m-1}\left(G\left(A^{\prime}\right)\right)=0$ and this says that

$$
f_{1} \cdots f_{m-1}(G(A)) \subseteq G\left(A_{1 m}\right) \oplus \cdots \oplus G\left(A_{m m}\right) .
$$

On the other hand, $f_{m}(G(A)) \subseteq G\left(A_{1}\right) \oplus \cdots \oplus G\left(A_{m-1}\right)+G(J)$. Thus

$$
f(G(A)) \subseteq\left(G\left(A_{1 m}\right) \oplus \cdots \oplus G\left(A_{m m}\right)\right)\left(G\left(A_{1}\right) \oplus \cdots \oplus G\left(A_{m-1}\right)+G(J)\right)=0
$$

since $A_{i m} J=A_{i m} A_{t}=0$ for all $i=1, \ldots, m, t=1, \ldots, m-1$.

Lemma 4.3. Let $A=A_{1} \oplus \cdots \oplus A_{m}+J$ be a minimal superalgebra. Then $S(A)$ contains a relatively free algebra of the variety determined by the T-ideal $\operatorname{Id}\left(G\left(A_{1}\right)\right) \cdots \operatorname{Id}\left(G\left(A_{m}\right)\right)$.

Proof. We proceed by induction on $m$. If $m=1$, the result follows from Lemma 2.2. Let $m>1$ and partition each of the sets $U$ and $V$ into three disjoint countable subsets

$$
U=\widetilde{U} \cup \bar{U} \cup \widehat{U}, \quad V=\widetilde{V} \cup \bar{V} \cup \widehat{V} .
$$


Hence the free supercommutative algebra $S=F[U, V]$ contains three isomorphic copies $\widetilde{S}=F[\widetilde{U}, \widetilde{V}], \bar{S}=F[\bar{U}, \bar{V}]$ and $\widehat{S}=F[\widehat{U}, \widehat{V}]$.

Let $C$ be the subalgebra of $A$ generated by $A_{1}, \ldots, A_{m-1}$ and by the elements $w_{12}, \ldots, w_{m-2, m-1}$. The algebra $C$ has an induced $\mathbb{Z}_{2}$-grading $C=C^{(0)} \oplus C^{(1)}$ and let $\left\{c_{1}^{(0)}, \ldots, c_{r_{1}}^{(0)}\right\}$ and $\left\{c_{1}^{(1)}, \ldots, c_{s_{1}}^{(1)}\right\}$ be homogeneous bases of $C^{(0)}$ and $C^{(1)}$, respectively.

By the inductive hypothesis the algebra $\widetilde{S}(C)$ contains a relatively free algebra $\widetilde{C}$ of the variety determined by the T-ideal $\operatorname{Id}\left(G\left(A_{1}\right)\right) \cdots \operatorname{Id}\left(G\left(A_{m-1}\right)\right)=$ $\operatorname{Id}\left(S\left(A_{1}\right)\right) \cdots \operatorname{Id}\left(S\left(A_{m-1}\right)\right)$. But, since $C$ is a minimal superalgebra, by Lemma 4.2 ,

$$
\operatorname{Id}(\widetilde{C})=\operatorname{Id}\left(S\left(A_{1}\right)\right) \cdots \operatorname{Id}\left(S\left(A_{m-1}\right)\right) \subseteq \operatorname{Id}(G(C)),
$$

and, by Lemma 2.2 $\operatorname{Id}(G(C))=\operatorname{Id}(\widetilde{S}(C))$. It follows that $\operatorname{Id}(\widetilde{C})=\operatorname{Id}(\widetilde{S}(C))$, i.e., $\widetilde{C}$ is the relatively free algebra of the variety $\operatorname{var}(G(C))$.

Now, for $k=1,2, \ldots$, set

$$
X_{k}=\sum_{j} c_{j}^{(0)} \otimes \widetilde{u}_{k j}+\sum_{j} c_{j}^{(1)} \otimes \widetilde{v}_{k j}
$$

where $\widetilde{u}_{k j} \in \widetilde{U}, \widetilde{v}_{k j} \in \widetilde{V}$ are distinct elements. Then by Lemma 2.2, we can suppose that $\widetilde{C}=F\left\{X_{1}, X_{2}, \ldots\right\}$ and $X_{1}, X_{2}, \ldots$ are free generators of the algebra $\widetilde{C}$.

Write $A_{m}=B=B^{(0)} \oplus B^{(1)}$ and take $\left\{b_{1}^{(0)}, \ldots, b_{r_{2}}^{(0)}\right\}$ as a basis of $B^{(0)}$ and $\left\{b_{1}^{(1)}, \ldots, b_{s_{2}}^{(1)}\right\}$ as a basis of $B^{(1)}$. For $k=1,2, \ldots$ set

$$
Y_{k}=\sum_{j} b_{j}^{(0)} \otimes \widehat{u}_{k j}+\sum_{j} b_{j}^{(1)} \otimes \widehat{v}_{k j}
$$

with $\widehat{u}_{k j} \in \widehat{U}, \widehat{v}_{k j} \in \widehat{V}$ distinct.

If $\widehat{B}$ denotes the relatively free algebra of the variety $\operatorname{var}\left(G\left(A_{m}\right)\right)$, then by Lemma 2.2, $\widehat{B}=F\left\{Y_{1}, Y_{2}, \ldots\right\}$ and $Y_{1}, Y_{2}, \ldots$ freely generate $\widehat{B}$ over $F$. Notice that $\widehat{B} \subseteq$ $\widehat{S}(B)$ and $\widetilde{C} \subseteq \widetilde{S}(C)$.

Now consider the subspace $A_{m-1} w_{m-1, m} A_{m}=A_{m-1, m}$ and let

$$
d_{1}^{(0)}, \ldots, d_{r_{3}}^{(0)} \in A_{m-1, m}^{(0)}, \quad d_{1}^{(1)}, \ldots, d_{s_{3}}^{(1)} \in A_{m-1, m}^{(1)}
$$

be a homogeneous basis. For $k=1,2, \ldots$ set

$$
Z_{k}=\sum_{j} d_{j}^{(0)} \otimes \bar{u}_{k j}+\sum_{j} d_{j}^{(1)} \otimes \bar{v}_{k j},
$$

with $\bar{u}_{k j} \in \bar{U}, \bar{v}_{k j} \in \bar{V}$ distinct.

We shall next prove that the elements $Z_{1}, Z_{2}, \ldots$ generate a free $(\widetilde{C}, \widehat{B})$-bimodule in $S(A)$.

Suppose to the contrary that there exists a non-trivial relation

$$
\sum_{i, j} a_{i j} Z_{j} b_{i j}=0
$$

where $a_{i j} \in \widetilde{C}, b_{i j} \in \widehat{B}$. Since any two distinct $Z_{i}$ and $Z_{j}$ depend on distinct sets of variables $\bar{u}_{k j}$ and $\bar{v}_{k j}$, from (4.2) we get a non-trivial relation of the type

$$
a_{1} Z_{1} b_{1}+\cdots+a_{n} Z_{1} b_{n}=0
$$


where the elements $a_{1}, \ldots, a_{n} \in \widetilde{C}$ are linearly independent and $b_{1}, \ldots, b_{n} \in \widehat{B}$ are all non-zero.

Recall that the elements $b_{1}, \ldots, b_{n}$ in the relation (4.3) are polynomials in the elements $Y_{1}, Y_{2}, \ldots$. We next claim that we may assume these polynomials to be multilinear.

In order to see this, suppose that $b_{1}, \ldots, b_{n}$ are polynomials in $Y_{1}, \ldots, Y_{k}$ and pick $\lambda_{1}, \ldots, \lambda_{k} \in F$. Define a map $\varphi: U \cup V \rightarrow S$ by setting

$$
\begin{aligned}
& \varphi\left(\widehat{u}_{i j}\right)=\lambda_{i} \widehat{u}_{i j}, \\
& \varphi\left(\widehat{v}_{i j}\right)=\lambda_{i} \widehat{v}_{i j},
\end{aligned}
$$

for all $i=1, \ldots, k$ and for all $j \geq 1$, and $\varphi$ is the identity map on the remaining elements of $U \cup V$. Then $\varphi$ extends to a homomorphism $\bar{\varphi}: A \otimes S \rightarrow A \otimes S$ such that

$$
\begin{gathered}
\bar{\varphi}\left(Y_{i}\right)= \begin{cases}\lambda_{i} Y_{i}, & \text { for } 1 \leq i \leq k, \\
Y_{i}, & \text { for } i \geq k+1,\end{cases} \\
\bar{\varphi}\left(X_{i}\right)=X_{i}, \bar{\varphi}\left(Z_{i}\right)=Z_{i}, \text { for } i \geq 1,
\end{gathered}
$$

and $\bar{\varphi}$ is the identity map on $A$. Since $F$ is infinite, a Vandermonde determinant argument shows that we may take $b_{1}, \ldots, b_{n}$ to be homogeneous in each variable $Y_{i}$.

In order to make $b_{1}, \ldots, b_{n}$ multilinear, we fix $Y_{i}, 1 \leq i \leq k$, and we construct an endomorphism $\psi: A \otimes S \rightarrow A \otimes S$ fixed on $A$ such that

$$
\psi\left(Y_{i}\right)=Y_{i}+Y_{k+1}, \psi\left(Y_{j}\right)=Y_{j}, \text { for } j \neq i,
$$

and

$$
\psi\left(X_{j}\right)=X_{j}, \psi\left(Z_{j}\right)=Z_{j}, \text { for all } j \geq 1 .
$$

Now in each $b_{i}$ appearing in (4.3) we replace $Y_{i}$ with $Y_{i}+Y_{k+1}$. A standard multilinearization process leads to the linearity of the polynomials $b_{1}, \ldots, b_{n}$ as claimed.

We have proved that in relation (4.3) we may take $b_{i}=b_{i}\left(Y_{1}, \ldots, Y_{k}\right), \quad i=$ $1, \ldots, n$, to be non-zero multilinear polynomials in $Y_{1}, \ldots, Y_{k}$.

Let $e_{m} \in A_{m}=B$ be the minimal graded idempotent such that $w_{m-1, m} e_{m}=$ $w_{m-1, m}$. We know, by Lemma 3.1, that $e_{m}=E_{\alpha \alpha} \in B^{(0)}$ is a diagonal matrix unit of the simple algebra $B$. By Lemma 4.1, there exists a non-zero monomial $p \in \widehat{F}[\widehat{U}, \widehat{V}]$ such that either $E \otimes p \in b_{1}(S(B))$ or $\left(E_{\alpha \alpha}-E_{\beta \beta}\right) \otimes p \in b_{1}(S(B))$ for any $\beta \neq \alpha$. Hence there exists a valuation $\varphi: Y_{i} \rightarrow h_{i} \otimes p_{i}, i=1, \ldots, k$, where $h_{i} \in B^{(0)} \cup B^{(1)}$ are homogeneous elements and $p_{i} \in \widehat{S}$ are monomials such that

$$
\varphi\left(b_{j}\right)=\left(\sum_{i, l} \lambda_{i l}^{j} E_{i l}\right) \otimes p
$$

with $\lambda_{\alpha \alpha}^{1} \neq 0$. Now, notice that since $a_{1}, \ldots, a_{n} \in \widetilde{C}$ are linearly independent and $\lambda_{\alpha \alpha}^{1} \neq 0$, the polynomial

$$
f=f\left(X_{1}, \ldots, X_{t}\right)=\sum_{j} \lambda_{\alpha \alpha}^{j} a_{j}
$$

is not an identity of $\widetilde{S}(C)$.

Since the elements $X_{i}, Y_{i}$ and $Z_{i}$ depend on disjoint sets of indeterminates, we obtain that for any evaluation $\eta: \widetilde{C} \rightarrow \widetilde{S}(C)$ there exists a homomorphism 
$\theta: A \otimes S \rightarrow A \otimes S$ which is the identity on $A$, such that

$$
\begin{gathered}
\theta\left(X_{i}\right)=\eta\left(X_{i}\right), i=1,2, \ldots, \\
\theta\left(Z_{1}\right)=w_{m-1, m} \otimes p^{\prime},
\end{gathered}
$$

where $p^{\prime}=1$ if $w_{m-1, m}$ is even, $p^{\prime} \in \bar{V}$ if $w_{m-1, m}$ is odd, and $\theta$ takes arbitrary values on $Y_{1}, \ldots, Y_{k}$ in $\widehat{S}(B)$. Let

$$
\theta\left(b_{j}\right)=\left(\sum_{i, l} \lambda_{i l}^{j} E_{i l}\right) \otimes p, j=1, \ldots, n .
$$

Then from (4.3) it follows that

$$
0=\theta\left(a_{1} Z_{1} b_{1}+\cdots+a_{n} Z_{1} b_{n}\right)=\sum_{j} \theta\left(a_{j}\right)\left(w_{m-1, m} \otimes p^{\prime}\right)\left(\left(\sum_{i, l} \lambda_{i l}^{j} E_{i l}\right) \otimes p\right) .
$$

Since $w_{m-1, m}=w_{m-1, m} E_{\alpha \alpha}$, we obtain

$$
0=\sum_{j} \theta\left(a_{j}\right)\left(w_{m-1, m} \otimes p^{\prime}\right)\left(\left(\sum_{l} \lambda_{\alpha l}^{j} E_{\alpha l}\right) \otimes p\right) .
$$

We now multiply the above relation by $E_{\alpha \alpha} \otimes 1$ on the right, and we get

$$
\begin{gathered}
0=\sum_{j} \theta\left(a_{j}\right)\left(w_{m-1, m} \otimes p^{\prime}\right)\left(\left(\lambda_{\alpha \alpha}^{j} E_{\alpha \alpha}\right) \otimes p\right)=\sum_{j} \lambda_{\alpha \alpha}^{j} \theta\left(a_{j}\right) w_{m-1, m} \otimes p^{\prime} p \\
=\theta\left(\sum_{j} \lambda_{\alpha \alpha}^{j} a_{j}\right) w_{m-1, m} \otimes p^{\prime} p=\eta\left(\sum_{j} \lambda_{\alpha \alpha}^{j} a_{j}\right) w_{m-1, m} \otimes p^{\prime} p .
\end{gathered}
$$

Since $\eta: \widetilde{C} \rightarrow \widetilde{S}(C)$ is an arbitrary evaluation and recalling the definition of $f$ given in (4.5), from (4.6) and (4.4) it follows that

$$
f(\widetilde{S}(C)) \cdot w_{m-1, m} \otimes p^{\prime} p=0 .
$$

If $m>2$, then, since $f$ is not an identity of $\widetilde{S}(C)$, by Lemma 4.1 $f(\widetilde{S}(C))$ contains the element $w_{1, m-1} \otimes p^{\prime \prime}$ for some monomial $p^{\prime \prime} \in \widetilde{S}$. Thus

$$
0 \neq w_{1, m} \otimes p^{\prime \prime} p^{\prime} p=\left(w_{1, m-1} \otimes p^{\prime \prime}\right)\left(w_{m-1, m} \otimes p^{\prime} p\right) \in f(\widetilde{S}(C)) \cdot w_{m-1, m} \otimes p^{\prime} p,
$$

and this is a contradiction.

In the case $m=2$, there exists a minimal idempotent $e_{1} \in A$ such that $e_{1} w_{1,2}=$ $w_{1,2}$ and, by Lemma 4.1, either $E_{1} \otimes p^{\prime \prime} \in f(\widetilde{S}(C))$ or $\left(e_{1}-e_{1}^{\prime}\right) \otimes p^{\prime \prime} \in f(\widetilde{S}(C))$, where $E_{1}$ is the unit element of $A_{1}$ and $e_{1}^{\prime} \neq e_{1}$ is a minimal idempotent of $A_{1}$. We get

$$
0 \neq w_{1,2} \otimes p^{\prime \prime} p^{\prime} p \in f(\widetilde{S}(C)) w_{1,2} \otimes p^{\prime} p
$$

a contradiction.

We have proved that the elements $Z_{1}, Z_{2}, \ldots$ generate a free $(\widetilde{C}, \widehat{B})$-bimodule in $S(A)$. By [24, Theorem 1] (see also [9] Theorem 3.9]) it follows that the subalgebra of $S(A)$ generated by $X_{i}+Y_{i}+Z_{i}, i=1,2, \ldots$, is the relatively free algebra determined by the T-ideal $\operatorname{Id}(S(C)) \operatorname{Id}(S(B))=\operatorname{Id}\left(S\left(A_{1}\right)\right) \cdots \operatorname{Id}\left(S\left(A_{m}\right)\right)$. By Lemma 2.2 the proof of the lemma is complete.

Let $A$ be a minimal superalgebra. Since by Lemma $2.2 G(A)$ and $S(A)$ satisfy the same identities, by combining Lemma 4.2 and Lemma 4.3 we obtain that $I d(G(A))=I d\left(G\left(A_{1}\right)\right) \cdots I d\left(G\left(A_{m}\right)\right)$. We record this in the following corollary. 
Corollary 4.4. If $A=A_{1} \oplus \cdots \oplus A_{m}+J$ is a minimal superalgebra, then $\operatorname{Id}(G(A))=$ $\operatorname{Id}\left(G\left(A_{1}\right)\right) \cdots \operatorname{Id}\left(G\left(A_{m}\right)\right)$.

\section{Products of verbally prime T-ideals}

Throughout this section $F$ will be an arbitrary field of characteristic zero, not necessarily algebraically closed.

Let $\mathcal{V}$ be a variety of associative algebras. Recall that $\mathcal{V}$ is minimal of exponent $d \geq 2$ if $\exp (\mathcal{V})=d$ and for every proper subvariety $\mathcal{U} \underset{\neq}{\mathcal{V}}$ we have that $\exp (\mathcal{U})<$ $d$. As a consequence of the results in section 3 and section 4 we first obtain a characterization of minimal varieties in terms of generating algebras and T-ideals. In fact we have

Theorem 5.1. Let $F$ be any field of characteristic zero and let $\mathcal{V}$ be a variety. If $\mathcal{V}$ is minimal of exponent $d \geq 2$, then $\mathcal{V}=\operatorname{var}(G(A))$ for some minimal superalgebra A. Hence there exist verbally prime T-ideals $I_{1}, \ldots, I_{m}$ such that $I d(\mathcal{V})=I_{1} \cdots I_{m}$.

Proof. Let $B$ be an algebra such that $\mathcal{V}=\operatorname{var}(B)$. If $\bar{F}$ is the algebraic closure of the field $F$, we form $\bar{B}=B \otimes_{F} \bar{F}$. Since the $n$th codimension $c_{n}(B)$ of $B$ over $F$ coincides with the $n$th codimension $c_{n}(\bar{B})$ of $\bar{B}$ over $\bar{F}$ (see for instance [11]), it follows that $\exp (\bar{B})=d$ over $\bar{F}$. By Lemma 3.4, $\operatorname{var}(\bar{B})$ contains the Grassmann envelope $G(A)$ of a minimal superalgebra $A$ and $\exp (\bar{B})=\exp (G(A))$. Since $\bar{B}$ as an $F$-algebra belongs to $\mathcal{V}$, it follows that $G(A) \in \mathcal{V}$. By the minimality of $\mathcal{V}$ we get that $\mathcal{V}=\operatorname{var}(G(A))$. Hence $\operatorname{Id}(\mathcal{V})=\operatorname{Id}(G(A))$ and by Corollary 4.4 $\operatorname{Id}(\mathcal{V})=I_{1} \cdots I_{m}$ with $I_{k}=\operatorname{Id}\left(G\left(A_{k}\right)\right), k=1, \ldots, m$, a verbally prime T-ideal since $A_{k}$ is a simple superalgebra of type 1$), 2$ ), or 3 ).

We now recall that a T-ideal $I$ of $F\langle X\rangle$ is verbally prime if $I_{1} I_{2} \subseteq I$ for any T-ideals $I_{1}, I_{2}$ implies that either $I_{1} \subseteq I$ or $I_{2} \subseteq I$. By a well-known result of Kemer ([21, Theorem 1.2]), a proper ideal $I$ is verbally prime if and only if $I=G(A)$ for some finite dimensional simple superalgebra $A$ of type 1),2), or 3 ) as described in section 3.

The previous result has a converse, as the following theorem shows

Theorem 5.2. If $I_{1}, \ldots, I_{m}$ are proper verbally prime T-ideals of $F\langle X\rangle$, then there exists a minimal superalgebra $A$ such that $I d(G(A))=I_{1} \cdots I_{m}$.

Proof. Let $A_{1}, \ldots, A_{m}$ be finite dimensional simple superalgebras of type 1), 2), or 3) such that $I_{j}=\operatorname{Id}\left(G\left(A_{j}\right)\right), j=1, \ldots, m$. For $j=1, \ldots, m$, we define subsets $Q_{j}$ as follows: if $A_{j}$ is of type 1 ) or 2 ), then we set $Q_{j}=\left\{1_{j}\right\}$ where $1_{j}$ is the unit element of $A_{j}$; if $A_{j}$ is of type 3), i.e., $A_{j}=M_{n_{j}}\left(F \oplus t_{i} F\right)$ with $t_{j}^{2}=1$, we set $Q_{j}=\left\{1_{j}, t_{j}\right\}$ where $1_{j}$ is the unit element of $M_{n_{j}}(F)$.

Let us now denote by $e_{i j}^{(\alpha)}$ the matrix units of the algebra $A_{\alpha}$ if $A_{\alpha}$ is of type 1) or 2). If $A_{\alpha}$ is of type 3 ), then we let $e_{i j}^{(\alpha)}$ be the matrix units of $A_{\alpha}^{(0)}$.

Now let $A_{i, i+1} \cong A_{i} e_{11}^{(i)} \otimes e_{11}^{(i+1)} A_{i+1}$ be the $\mathbb{Z}_{2}$-graded irreducible $\left(A_{i}, A_{i+1}\right)$ bimodule with even generator $w_{i, i+1}$ satisfying the equalities

$$
w_{i, i+1}=e_{11}^{(i)} w_{i, i+1}=w_{i, i+1} e_{11}^{(i+1)} .
$$


More generally, for any $j \geq i+2$ and for any $q_{i+1} \in Q_{i+1}, \ldots, q_{j-1} \in Q_{j-1}$, let $A_{i j}\left(q_{i+1}, \ldots, q_{j-1}\right)$ be the $\mathbb{Z}_{2}$-graded irreducible $\left(A_{i}, A_{j}\right)$-bimodule with homogeneous generator $w_{i, j}\left(q_{i+1}, \ldots, q_{j-1}\right)$ satisfying the equalities

$$
w_{i j}\left(q_{i+1}, \ldots, q_{j-1}\right)=e_{11}^{(i)} w_{i j}\left(q_{i+1}, \ldots, q_{j-1}\right)=w_{i j}\left(q_{i+1}, \ldots, q_{j-1}\right) e_{11}^{(j)} .
$$

Note that the degree of the element $w_{i j}\left(q_{i+1}, \ldots, q_{j-1}\right)$ in the $\mathbb{Z}_{2}$-grading depends on $q_{i+1}, \ldots, q_{j-1}$; in fact $w_{i j}\left(q_{i+1}, \ldots, q_{j-1}\right)$ is even or odd according to whether the number of $q_{\alpha}=t_{\alpha} \in Q_{\alpha}, i+1 \leq \alpha \leq j-1$, is even or odd, respectively.

Now define $A_{11}=A_{1}, \ldots, A_{m m}=A_{m}$ and

$$
A_{i j}=\bigoplus_{q_{i+1} \in Q_{i+1}, \ldots, q_{j-1} \in Q_{j-1}} A_{i j}\left(q_{i+1}, \ldots, q_{j-1}\right)
$$

Then set

$$
A=\bigoplus_{1 \leq i \leq j \leq m} A_{i j}
$$

We make $A$ into an algebra by defining the following multiplication. If $a, b \in A_{i i}$ for some $i$, then $a b$ is the ordinary product in the algebra $A_{i i}$. For any $1 \leq i \leq j \leq$ $m, 1 \leq k \leq l \leq m$ with $j \neq k$ we set $A_{i j} A_{k l}=0$. We now define a multiplication $a b$ where $a \in A_{i j}, b \in A_{j k}$ as follows: from (5.1) it follows that the elements

$$
q_{i} e_{\alpha 1}^{(i)} w_{i j}\left(q_{i+1}, \ldots, q_{j-1}\right) q_{j} e_{1 \beta}^{(j)}
$$

with $q_{i} \in Q_{i}, \ldots, q_{j} \in Q_{j}, 1 \leq \alpha \leq n_{i}, 1 \leq \beta \leq n_{j}$, form a linear basis of $A_{i j}$. Denote for short $\bar{q}_{i j}=\left(q_{i+1}, \ldots, q_{j-1}\right)$ and define

$$
\begin{gathered}
q_{i} e_{\alpha 1}^{(i)} w_{i j}\left(\bar{q}_{i j}\right) q_{j} e_{1 \beta}^{(j)} \cdot q_{j}^{\prime} e_{\gamma 1}^{(j)} w_{j k}\left(\bar{q}_{j k}^{\prime}\right) q_{k}^{\prime} e_{1 \delta}^{(k)} \\
= \begin{cases}q_{i} e_{\alpha 1}^{(i)} w_{i k}\left(\bar{q}_{i j}, q_{j} q_{j}^{\prime}, \bar{q}_{j k}^{\prime}\right) q_{k}^{\prime} e_{1 \delta}^{(k)}, & \text { if } \beta=\gamma, \\
0, & \text { otherwise. }\end{cases}
\end{gathered}
$$

With this definition $A$ becomes an associative finite dimensional superalgebra with maximal semisimple subalgebra $A_{1} \oplus \cdots \oplus A_{m}$ and Jacobson radical

$$
J=\bigoplus_{1 \leq i<j \leq m} A_{i j}
$$

Clearly $A$ is a minimal superalgebra and, by Corollary 4.4, we have that $\operatorname{Id}(G(A))=$ $I_{1} \cdots I_{m}$.

In the next theorem we generalize the defining property of a verbally prime T-ideal to products of verbally prime T-ideals.

Theorem 5.3. Let $I_{1}, \ldots, I_{m}$ be verbally prime $T$-ideals and set $I=I_{1} \cdots I_{m}$. If $P, Q$ are $T$-ideals such that $P Q \subseteq I$, then either $P \subseteq I$ or $Q \subseteq I$ or there exists $1 \leq k \leq m-1$ such that $P \subseteq I_{1} \cdots I_{k}$ and $Q \subseteq I_{k+1} \cdots I_{m}$.

Proof. Suppose that $P \nsubseteq I$ and $Q \nsubseteq I$. Since $I$ is the product of verbally prime T-ideals, by Theorem 5.2 there exists a minimal superalgebra $A$ such that $I=$ $I d(G(A))$. Moreover if $A=A_{s s}+J$ with $A_{s s}=A_{1} \oplus \cdots \oplus A_{m}$ a maximal semisimple $\mathbb{Z}_{2}$-stable subalgebra, then $I_{k}=I d\left(G\left(A_{k}\right)\right)$ for $k=1, \ldots, m$.

Since $P \nsubseteq I$, there exists a smallest integer $k \in\{1, \ldots, m\}$ such that $P \nsubseteq$ $I_{1} \cdots I_{k}$. If $Q \subseteq I_{k} \cdots I_{m}$, then we are done. Therefore we may assume that $Q \nsubseteq I_{k} \cdots I_{m}$. 
Let $f \in P \backslash I_{1} \cdots I_{k}$ and $g \in Q \backslash I_{k} \cdots I_{m}$ be multilinear polynomials in disjoint sets of variables. Since $f g \in P Q \subseteq I$, we shall reach a contradiction by proving that $f g \notin I_{1} \cdots I_{m}=I=I d(G(A))$.

Clearly $m \geq 2$. Suppose first that $k \neq 1, m-1$. Let $B$ be the subalgebra of $A$ generated by $A_{1}, \ldots, A_{k}, w_{12}, \ldots, w_{k-1, k}$ and let $C$ be the subalgebra generated by $A_{k}, \ldots, A_{m}, w_{k, k+1}, \ldots, w_{m-1, m}$. Since $B$ and $C$ are both minimal superalgebras, by Lemma 4.1] there exist monomials $p, q \in S=F[U, V]$ such that

$$
w_{1 k} \otimes p \in f(S(B)) \quad \text { and } \quad w_{k m} \otimes q \in g(S(C)) .
$$

Since we may clearly assume that $p q \neq 0$, then $f(S(B)) f(S(C)) \neq 0$, i.e., $f g$ is not an identity of $S(A)$. Since by Lemma $2.2 S(A)$ and $G(A)$ have the same identities, we obtain that $f g \notin \operatorname{Id}(G(A))$ and we are done in this case.

Suppose now that $k=1$. Then as above, invoking Lemma 4.1, there exist monomials $p, q \in S=F[U, V]$ such that

$$
w_{1 m} \otimes q \in g(S(A))
$$

and either $\left(e_{1}-e_{1}^{\prime}\right) \otimes p \in f\left(S\left(A_{1}\right)\right)$ where $e_{1} w_{1 m}=w_{1 m}, e_{1}^{\prime} w_{1 m}=0$ or $E \otimes p \in$ $f\left(S\left(A_{1}\right)\right)$ with $E$ the unit element of $A_{1}$. Again, $f\left(S\left(A_{1}\right)\right) g(S(A)) \neq 0$ and $f g$ is not an identity of $S(A)$. The case $k=m$ is similar.

The argument of the previous theorem can be applied in order to prove the following

Proposition 5.4. Let $I_{1}, \ldots, I_{m}$ be proper verbally prime $T$-ideals and $m \geq 2$. If for some T-ideals $P, Q$ we have $[P, Q] \subseteq I_{1} \cdots I_{m}$, then $P Q+Q P \subseteq I_{1} \cdots I_{m}$.

Proof. If either $P$ or $Q$ is contained in $I=I_{1} \cdots I_{m}$, then the conclusion is clearly true. Hence we may assume that $P \nsubseteq I$ and $Q \nsubseteq I$. But then by the same arguments as in the previous theorem we obtain that $P Q \subseteq I$ and $Q P \subseteq I$ as desired.

We conclude this section with some examples.

We first remark that any minimal superalgebra $A$ can be realized as a subalgebra of some block triangular matrix algebra with suitable $\mathbb{Z}_{2}$-grading. For instance, if $A_{s s}=A_{1} \oplus \cdots \oplus A_{m}$ and for all $j=1, \ldots, m, A_{j} \cong M_{d_{j}}(F)$ is an ordinary matrix algebra with trivial $\mathbb{Z}_{2}$-grading, then (see 14 or $[13]$ )

$$
A \cong\left(\begin{array}{cccc}
M_{d_{1}}(F) & & & * \\
0 & \ddots & & \\
\vdots & & & \\
0 & \cdots & 0 & M_{d_{m}}(F)
\end{array}\right),
$$

the algebra of upper block triangular matrices with trivial $\mathbb{Z}_{2}$-grading.

In general, if two minimal superalgebra $A$ and $B$ have isomorphic maximal semisimple $\mathbb{Z}_{2}$-stable subalgebras with simple components of type 1) or 2), then $A \cong B$ as non-graded algebras. Anyway they might be equipped with distinct $\mathbb{Z}_{2}$-gradings and might be non-isomorphic as superalgebras. Nevertheless their Grassmann envelopes satisfy the same identities.

In case simple superalgebras of type 3) appear, then the corresponding minimal superalgebras may not even be isomorphic in the ordinary sense. Put in another way, since minimal superalgebras correspond to products of verbally prime 
T-ideals, to a given product of verbally prime T-ideals can correspond distinct non-isomorphic minimal superalgebras. We give an example to illustrate this fact.

Set $M_{k}(F)=M$ and let

$$
R_{1}=\left(\begin{array}{cccc}
M & 0 & M & M \\
0 & M & M & M \\
0 & 0 & M & 0 \\
0 & 0 & 0 & M
\end{array}\right)
$$

with grading

$$
\left(\left(\begin{array}{cccc}
A & 0 & C & D \\
0 & A & D & C \\
0 & 0 & B & 0 \\
0 & 0 & 0 & B
\end{array}\right),\left(\begin{array}{cccc}
A^{\prime} & 0 & C^{\prime} & D^{\prime} \\
0 & -A^{\prime} & -D^{\prime} & -C^{\prime} \\
0 & 0 & B^{\prime} & 0 \\
0 & 0 & 0 & -B^{\prime}
\end{array}\right)\right)
$$

let

$$
R_{2}=\left(\begin{array}{cccc}
M & 0 & 0 & M \\
0 & M & M & 0 \\
0 & 0 & M & 0 \\
0 & 0 & 0 & M
\end{array}\right)
$$

with grading

$$
\left(\left(\begin{array}{cccc}
A & 0 & 0 & C \\
0 & A & C & 0 \\
0 & 0 & B & 0 \\
0 & 0 & 0 & B
\end{array}\right),\left(\begin{array}{cccc}
A^{\prime} & 0 & 0 & D \\
0 & -A^{\prime} & -D & 0 \\
0 & 0 & B^{\prime} & 0 \\
0 & 0 & 0 & -B^{\prime}
\end{array}\right)\right)
$$

and let

$$
R_{3}=\left(\begin{array}{cccc}
M & 0 & M & M \\
0 & M & 0 & M \\
0 & 0 & M & 0 \\
0 & 0 & 0 & M
\end{array}\right)
$$

with grading

$$
\left(\left(\begin{array}{cccc}
A & 0 & C & D \\
0 & A & 0 & C \\
0 & 0 & B & 0 \\
0 & 0 & 0 & B
\end{array}\right),\left(\begin{array}{cccc}
A^{\prime} & 0 & C^{\prime} & D^{\prime} \\
0 & -A^{\prime} & 0 & -C^{\prime} \\
0 & 0 & B^{\prime} & 0 \\
0 & 0 & 0 & -B^{\prime}
\end{array}\right)\right)
$$

where $A, B, C, D, A^{\prime}, B^{\prime}, C^{\prime}, D^{\prime} \in M_{k}(F)$. Then $R_{i} \neq R_{j}$ for all $\leq i<j \leq 3$ whereas

$$
I d\left(G\left(R_{1}\right)\right)=\operatorname{Id}\left(G\left(R_{2}\right)\right)=\operatorname{Id}\left(G\left(R_{2}\right)\right)=\operatorname{Id}\left(M_{k}(G)\right)^{2} .
$$

\section{6. $S_{n}$-REPRESENTATIONS AND PI-ALGEBRAS}

In this section we recall the methods of representation theory of the symmetric group $S_{n}$ that we shall need to apply in the following section.

For every $n \geq 1$, let

$$
P_{n}=\operatorname{Span}_{F}\left\{x_{\sigma(1)} \cdots x_{\sigma(n)} \mid \sigma \in S_{n}\right\}
$$

be the subspace of $F\langle X\rangle$ consisting of all multilinear polynomials in the variables $x_{1}, \ldots, x_{n}$. The symmetric group $S_{n}$ acts naturally on the left of $P_{n}$ as follows: for $\sigma \in S_{n}$ and $f\left(x_{1}, \ldots, x_{n}\right) \in P_{n}$,

$$
\sigma f\left(x_{1}, \ldots, x_{n}\right)=f\left(x_{\sigma(1)}, \ldots, x_{\sigma(n)}\right) .
$$


Hence $P_{n}$ is an $S_{n}$-module and, for any PI-algebra $A$, the subspace $P_{n} \cap \operatorname{Id}(A)$ is $S_{n}$-invariant. We let

$$
P_{n}(A)=\frac{P_{n}}{P_{n} \cap I d(A)}
$$

be the induced $S_{n}$-module; then the $S_{n}$-character of $P_{n}(A)$ is called the $n$-th cocharacter of the algebra $A$ and is denoted $\chi_{n}(A)$. Since char $F=0$, every $S_{n^{-}}$ representation decomposes into the direct sum of irreducibles. Recall that there exists a one-to-one correspondence between irreducible $S_{n}$-characters and partitions on $n$. If $\lambda \vdash n$ is a partition of $n$, we denote by $\chi_{\lambda}$ the corresponding $S_{n}$-character. Then the $n$-th cocharacter of the PI-algebra $A$ decomposes as

$$
\chi_{n}(A)=\sum_{\lambda \vdash n} m_{\lambda} \chi_{\lambda},
$$

where $m_{\lambda} \geq 0$ is the multiplicity of the character $\chi_{\lambda}$ in $\chi_{n}(A)$. The study of the sequence of cocharacters of a PI-algebra is very useful for investigating the asymptotic behaviour of the codimensions $c_{n}(A), n \rightarrow \infty$.

For a partition $\lambda \vdash n$ let $D_{\lambda}$ be the corresponding Young diagram; usually we shall identify $\lambda$ with its diagram. By filling up the boxes of the diagram of $\lambda$ with the integers $1, \ldots, n$, one obtains a Young tableau $T_{\lambda}$. Let $e_{T_{\lambda}}$ be the essential idempotent of $F S_{n}$ corresponding to $T_{\lambda}$. Recall that

$$
e_{T_{\lambda}}=\sum_{\substack{\sigma \in R_{T_{\lambda}} \\ \tau \in C_{T_{\lambda}}}}(\operatorname{sgn} \tau) \sigma \tau
$$

where $R_{T_{\lambda}}, C_{T_{\lambda}}$ are the subgroups of $S_{n}$ preserving the rows and the columns of $T_{\lambda}$, respectively.

We define a partial order on the set of partitions as follows: given two partitions $\lambda=\left(\lambda_{1}, \ldots, \lambda_{k}\right)$ and $\mu=\left(\mu_{1}, \ldots, \mu_{t}\right)$, we say that $\lambda \geq \mu$ if $k \geq t$ and $\lambda_{1} \geq$ $\mu_{1}, \ldots, \lambda_{k} \geq \mu_{k}$. Notice that in the language of diagrams, $\lambda \geq \mu$ means that $D_{\mu}$ is a subdiagram of $D_{\lambda}$.

Given integers $l, d, t \geq 0$ denote by

$$
h(l, d, t)=(\underbrace{l+t, \ldots, l+t}_{d}, \underbrace{l, \ldots, l}_{t})
$$

the partition whose Young diagram has the first $d$ rows of length $l+t$ and the remaining $t$ rows of length $l$. It is clear that the corresponding diagram is hook shaped (see the following picture):

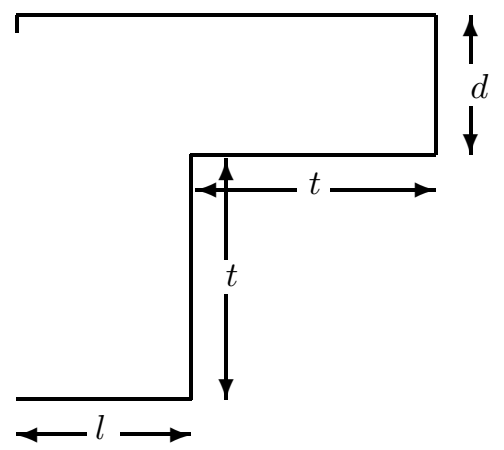


We also define an infinite hook $H(l, d)$ as the union of all $h(l, d, t), t \geq 1$,

$$
H(l, d)=\bigcup_{t \geq 1} h(l, d, t)
$$

(see the following picture):

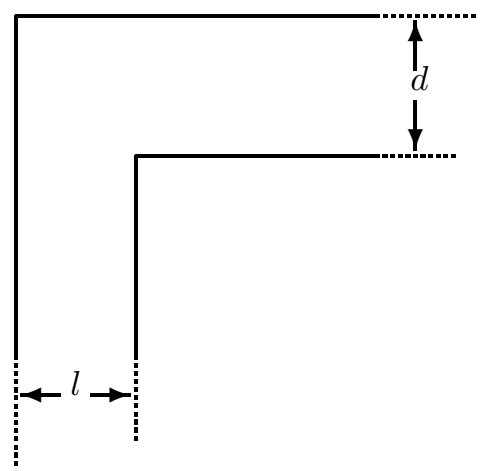

For a PI-algebra $A$ with cocharacter $\chi_{n}(A)=\sum_{\lambda \vdash n} m_{\lambda} \chi_{\lambda}$ we say that

$$
\chi_{n}(A) \subseteq H(l, d)
$$

if for all $n \geq 1$ there exists $t=t(n) \geq 1$ such that $\lambda \leq h(l, d, t)$ for all $\lambda \vdash n$ with $m_{\lambda} \neq 0$.

The importance of hooks in PI-theory is due to a theorem of Amitsur-Regev (1]) stating that for any PI-algebra $A$ there exist two integers $d, l \geq 0$ such that $\chi_{n}(A) \subseteq H(l, d)$. It turns out that for some PI-algebras it is possible to determine a minimal hook containing the cocharacter. Below we state a result that we shall need in the next section.

Lemma 6.1 ([2]). Let $B=B^{(0)} \oplus B^{(1)}$ be a finite dimensional simple superalgebra over $F$. If $\operatorname{dim}_{F} B^{(0)}=d, \operatorname{dim}_{F} B^{(1)}=l$, then $\chi_{n}(G(B)) \subseteq H(l, d)$ for all $n \geq 1$.

\section{Classifying minimal varieties of exponential growth}

In this section we shall classify all minimal varieties of algebras of exponent $\geq 2$. Drensky in 8 conjectured that a variety $\mathcal{V}$ is minimal if and only if its T-ideal of identities $\operatorname{Id}(\mathcal{V})$ is a product of verbally prime T-ideals. Here we shall prove this conjecture.

Concerning past results, from Kemer's work 20] it turns out that the Grassmann algebra $G$ and the algebra of $2 \times 2$ upper triangular matrices $U T_{2}$ generate the only two minimal varieties of exponent 2 . In [7] and [8] Drensky proved that any polynomial of the type $\left[x_{1}, x_{2}\right] \cdots\left[x_{2 n-1}, x_{2 n}\right]$ or $\left[x_{1}, x_{2}, x_{3}\right] \cdots\left[x_{3 n-2}, x_{3 n-1}, x_{3 n}\right]$ determines a minimal variety. Another example was given in [30] by proving that the polynomial $\left[x_{1}, x_{2}, x_{3}\right]\left[x_{4}, x_{5}\right]$ defines a minimal variety. In [16] all minimal varieties of exponent 3 were classified.

Recall that a variety is of finite basic rank if it is generated by a finitely generated algebra. In [14] the authors classified all minimal varieties of finite basic rank by proving Drensky's conjecture for such varieties.

Recall that we have already proved in Theorem 5.1 that if $\mathcal{V}$ is a minimal variety of exponent $\geq 2$, then there exist verbally prime T-ideals $I_{1}, \ldots, I_{m}$ such that 
$\operatorname{Id}(\mathcal{V})=I_{1} \cdots I_{m}$. In order to prove the other direction of Drensky's conjecture we shall next compare products of verbally prime T-ideals.

Recall that if $I$ is a T-ideal and $I=I d(\mathcal{V})$ for some variety of algebras $\mathcal{V}$, then $\exp (I)=\exp (\mathcal{V})$. We start by comparing the exponent of verbally prime T-ideals.

Lemma 7.1. Let $I \subseteq Q$ be two verbally prime T-ideals. Then $\exp (I)=\exp (Q)$ if and only if $I=Q$.

Proof. Let $I=I d(G(A)), Q=I d(G(B))$ where $A=A^{(0)} \oplus A^{(1)}, B=B^{(0)} \oplus B^{(1)}$ are finite dimensional simple superalgebras. Denote $d=\operatorname{dim} A^{(0)}, l=\operatorname{dim} A^{(1)}, s=$ $\operatorname{dim} B^{(0)}, r=\operatorname{dim} B^{(1)}$. Then $\exp (I)=d+l$ and $\exp (Q)=s+r$ (see, for example, [12]) and, by Lemma 6.1

$$
\chi_{n}(G(A)) \subseteq H(l, d) \quad \text { and } \quad \chi_{n}(G(B)) \subseteq H(r, s) .
$$

Since $I \subseteq Q$ then $\chi_{n}(G(B)) \subseteq \chi_{n}(G(A)) \subseteq H(l, d)$ and, by [12, Lemma 11], this is possible only if $r \leq l$ and $s \leq d$.

Suppose that $\exp (I)=\exp (Q)$. Then we obtain $r=l$ and $s=d$, i.e., $\operatorname{dim} A^{(0)}=$ $\operatorname{dim} B^{(0)}, \operatorname{dim} A^{(1)}=\operatorname{dim} B^{(1)}$. Now, if, say, $A=M_{n}(F)$ is a simple superalgebra of type 1) (see section 3), then $A^{(1)}=0$. But then also $B^{(1)}=0$ and $B$ is of type 1) as well. Thus $A \cong B$ in this case. If $A=M_{n}(F \oplus t F)$ is of type 3$)$, then $\operatorname{dim} A=2 n^{2}$. Since all simple superalgebras of type 1) or 2) have order a square, it follows that $B$ must also be of type 3 ); hence $A \cong B$ also in this case. Finally if $A=M_{p, q}$ is of type 2), then $B$ must also be of type 2). Say $B=M_{a, b}$. Since $\operatorname{dim} A^{(0)}=p^{2}+q^{2}=a^{2}+b^{2}=\operatorname{dim} B^{(0)}$ and $\operatorname{dim} A^{(1)}=2 p q=2 a b=\operatorname{dim} B^{(1)}$, we obtain $a=p, b=q$ and $A \cong B$ follows. This completes the proof of the lemma.

In the next lemma we analyze the more general situation when a verbally prime T-ideal is contained in a product of such T-ideals

Lemma 7.2. Let $m \geq 2$ and let $I_{1}, \ldots, I_{m}, Q$ be proper verbally prime T-ideals such that $Q \subseteq I_{1} \cdots I_{m}$. Then $\exp (Q)>\exp \left(I_{1} \cdots I_{m}\right)$.

Proof. Clearly $\exp (Q) \geq \exp \left(I_{1} \cdots I_{m}\right)$. Hence we only need to show that $\exp (Q) \neq$ $\exp \left(I_{1} \cdots I_{m}\right)$. Suppose to the contrary that $\exp (Q)=\exp \left(I_{1} \cdots I_{m}\right)$. By Theorem 5.2 there exists a minimal superalgebra $A=A^{(0)} \oplus A^{(1)}$ with maximal semisimple $\mathbb{Z}_{2}$-stable subalgebra $A_{s s}=A_{1} \oplus \cdots \oplus A_{m}$ such that $I=I_{1} \cdots I_{m}=I d(G(A))$ and $I_{j}=I d\left(G\left(A_{j}\right)\right), j=1, \ldots, m$. Also, let $B$ be the finite dimensional simple superalgebra such that $I d(B)=Q$. By Lemma $6.1 \chi_{n}(G(B)) \subseteq H(l, d)$, where $d=\operatorname{dim} B^{(0)}$ and $l=\operatorname{dim} B^{(1)}$; moreover by [12], $\exp (Q)=\exp (G(B))=d+l$. Since by hypothesis $\operatorname{Id}(G(B))=Q \subseteq I=I d(G(A))$, then

$$
\chi_{n}(G(A)) \subseteq H(l, d) .
$$

Moreover by assumption, $\exp (G(A))=\exp (G(B))=d+l$. For $i=1, \ldots, m$, denote $d_{i}=\operatorname{dim} A_{i}^{(0)}, l_{i}=\operatorname{dim} A_{i}^{(1)}$ and $l^{\prime}=l_{1}+\cdots+l_{m}, d^{\prime}=d_{1}+\cdots+d_{m}$.

For every $n \geq 1$, let $W_{n+m-1}$ be the space of all multilinear polynomials in the variables $x_{1}, \ldots, x_{n}, y_{1}, \ldots, y_{m-1}$ of the type

$$
f\left(x_{1}, \ldots, x_{n}, y_{1}, \ldots, y_{m-1}\right)=f_{1} y_{1} f_{2} \cdots f_{m-1} y_{m-1} f_{m}
$$

where $f_{1}, \ldots, f_{m}$ are polynomials in the $x_{i}$ 's. The permutation action of $S_{n}$ on the variables $x_{1}, \ldots, x_{n}$ turns $W_{n+m-1}$ into an $S_{n}$-module. Since $A$ is a minimal 
superalgebra, $A_{1} J \cdots J A_{m} \neq 0$, where $J=J(A)$ and we can apply [12, Lemma 15]. Therefore for any positive integer $t \geq 2 \operatorname{dim} A$, there exists $n$ such that

$$
l^{\prime} d^{\prime}+(2 t-s)\left(l^{\prime}+d^{\prime}\right) \leq n \leq l^{\prime} d^{\prime}+2 t\left(l^{\prime}+d^{\prime}\right), \quad s=4 \operatorname{dim} A,
$$

and a polynomial $f \in W_{n+m-1}$ which is not an identity of $G(A)$. Moreover $F S_{n} f=$ $M$, the $S_{n}$-module generated by $f$, is irreducible with character $\chi_{\lambda}$, where

$$
h\left(l^{\prime}, d^{\prime}, 2 t-s\right) \leq \lambda \leq h\left(l^{\prime}, d^{\prime}, 2 t\right) .
$$

If we now regard $W_{n+m-1}$ as an $S_{n+m-1}$-module (via the permutation action), then, by the Littlewood-Richardson rule ([19, Theorem 2.8.13]), any irreducible component of $M \uparrow S_{n+m-1}$, the $S_{n+m-1}$-module generated by $f$, is associated to a partition $\mu \vdash n+m-1$ with $\mu \geq \lambda$. But then, since $l+d=l^{\prime}+d^{\prime}$, from (7.1) we obtain that $l^{\prime}=l$ and $d^{\prime}=d$.

We shall reach a contradiction by proving that $\chi_{n}(G(A)) \nsubseteq H(l, d)$. Now, in 12 . Lemma 15] it was also shown that there exists an evaluation

$$
\varphi: x_{1}, \ldots, x_{n} \rightarrow G\left(A_{1} \oplus \cdots \oplus A_{m}\right), y_{1}, \ldots, y_{m-1} \rightarrow G(J)
$$

such that $\varphi(f) \neq 0$ and $\varphi(f) \in A_{1} J A_{2} J \cdots J A_{m}$. Since $F S_{n} f=M$ is irreducible with character $\chi_{\lambda}$, there exists a Young tableau $T_{\lambda}$ such that

$$
e_{T_{\lambda}} f=\gamma f
$$

for some $0 \neq \gamma \in F$. We rename the variables $y_{1}=x_{n+1}, \ldots, y_{m-1}=x_{n+m-1}$ and we construct a partition $\mu \vdash n+m-1$ and a corresponding Young tableau $T_{\mu}$ by the following rules: we let $\mu_{i}=\lambda_{i}$ for all $i \neq d+1$ and $\mu_{d+1}=\lambda_{d+1}+m-1=l+m-1$. Since $D_{\lambda}$ is a subdiagram of $D_{\mu}$ we let $T_{\mu}$ be the tableau containing the tableau $T_{\lambda}$ and with the entries $n+1, \ldots, n+m-1$ in the boxes $(d+1, l+1), \ldots,(d+1, l+m-1)$, respectively.

We wish to show that $e_{T_{\mu}} f \notin I d(G(A))$. To this end, since $T_{\lambda}$ is a subtableau of $T_{\mu}$, then, by considering the canonical embedding $S_{n} \subseteq S_{n+m-1}$, we get that $C_{T_{\lambda}} \subseteq C_{T_{\mu}}$ and $R_{T_{\lambda}} \subseteq R_{T_{\mu}}$.

Let $\varphi$ be the above evaluation of $f$; notice that from the multiplication rules of $A$, it easily follows that for any permutation $\rho \in S_{n+m-1}, \varphi(\rho f)$ is non-zero only if $\rho(n+1)=n+1, \ldots, \rho(n+m-1)=n+m-1$. On the other hand, if $\rho \in C_{T_{\mu}}$ and $\rho(n+1)=n+1, \ldots, \rho(n+m-1)=n+m-1$, then $\rho \in C_{T_{\lambda}}$. Hence

$$
\varphi\left(\sum_{\tau \in C_{T_{\mu}}}(\operatorname{sgn} \tau) \tau f\right)=\varphi\left(\sum_{\rho \in C_{T_{\lambda}}}(\operatorname{sgn} \rho) \rho f\right) .
$$

If we apply the same argument to $R_{T_{\lambda}}, R_{T_{\mu}}$ and $g=\left(\sum_{\rho \in C_{T_{\lambda}}}(\operatorname{sgn} \rho) \rho f\right.$, we obtain

$$
\varphi\left(e_{T_{\mu}} f\right)=\varphi\left(\sum_{\sigma \in R_{T_{\lambda}}} \sigma g\right)=\varphi\left(e_{T_{\lambda}} f\right)=\gamma \varphi(f) \neq 0 .
$$

This proves that $e_{T_{\mu}} f \notin I d(G(A))$ and, since $\mu \notin H(l, d)$, we get $\chi_{n}(G(A)) \nsubseteq$ $H(l, d)$. With this contradiction the proof is complete.

We next generalize Lemma 7.2 to the case of several factors.

Lemma 7.3. Let $I_{1}, \ldots, I_{m}, Q_{1}, \ldots, Q_{n}$ be proper verbally prime $T$-ideals such that

$$
Q_{1} \cdots Q_{n} \subseteq I_{1} \cdots I_{m}
$$

Then either $\exp \left(Q_{1} \cdots Q_{n}\right)>\exp \left(I_{1} \cdots I_{m}\right) \quad$ or $m=n$ and $I_{1}=Q_{1}, \ldots, I_{m}=$ $Q_{m}$. 
Proof. The case $n=1$ was settled in Lemma 7.2 Suppose $n \geq 2$ and denote $Q^{\prime}=$ $Q_{2} \cdots Q_{n}$. If either $Q_{1}$ or $Q^{\prime}$ is contained in $I_{1} \cdots I_{m}$, then, since by [4] $\exp \left(Q_{1} Q^{\prime}\right)=$ $\exp \left(Q_{1}\right)+\exp \left(Q^{\prime}\right)$, we obtain by induction on $n$ that $\exp \left(Q_{1} Q^{\prime}\right)>\exp \left(I_{1} \cdots I_{m}\right)$ and we are done in this case. Therefore we may assume that $Q_{1}, Q^{\prime} \nsubseteq I_{1} \cdots I_{m}$.

By Theorem 5.3, there exists $k \geq 1$ such that $Q_{1} \subseteq I_{1} \cdots I_{k}$ and $Q^{\prime} \subseteq I_{k+1} \cdots I_{m}$. Now, if $k>1$, by Lemma 7.2, $\exp \left(Q_{1}\right)>\exp \left(I_{1} \cdots I_{k}\right)$; hence

$$
\begin{gathered}
\exp \left(Q_{1} Q^{\prime}\right)=\exp \left(Q_{1}\right)+\exp \left(Q^{\prime}\right) \\
>\exp \left(I_{1} \cdots I_{k}\right)+\exp \left(I_{k+1} \cdots I_{m}\right)=\exp \left(I_{1} \cdots I_{m}\right)
\end{gathered}
$$

and we are done. Therefore we may assume that $k=1$, i.e., $Q_{1} \subseteq I_{1}$. A repeated application of this process leads to $m=n$ and $Q_{1} \subseteq I_{1}, \ldots, Q_{m} \subseteq I_{m}$. The conclusion of the lemma now follows from Lemma 7.1

As an immediate consequence of the above lemma we get the following

Corollary 7.4. Let $I_{1}, \ldots, I_{m}, Q_{1}, \ldots, Q_{n}$ be proper verbally prime $T$-ideals. Then

$$
I_{1} \cdots I_{m}=Q_{1} \cdots Q_{n}
$$

if and only if $m=n$ and $I_{1}=Q_{1}, \ldots, I_{m}=Q_{m}$.

Corollary 7.4 says that all verbally prime T-ideals of the free algebra $F\langle X\rangle$ generate a free semigroup. But this result can also be deduced from [6. Theorem 7], where it was proved that the semigroup of all non-zero T-ideals of $F\langle X\rangle$ is free. In fact, any verbally prime T-ideal by definition cannot be decomposed into the product of two T-ideals properly contained in it.

We are now in a position to state some interesting consequences. In the next theorem the equivalence of 1) and 2) is Drensky's conjecture.

Theorem 7.5. Let $\mathcal{V}$ be a variety of algebras over a field $F$ of characteristic zero such that $\exp (\mathcal{V}) \geq 2$. Then the following properties are equivalent:

(1) $\mathcal{V}$ is a minimal variety of exponent $d$;

(2) $\operatorname{Id}(\mathcal{V})$ is a product of verbally prime T-ideals;

(3) $\mathcal{V}=\operatorname{var}(G(A))$, for some minimal superalgebra $A$ such that $\operatorname{dim} A_{s s}=d$.

Proof. Theorem 5.1 says that 1) implies 2) and 3). Also, by Theorem 5.2 and Corollary 4.4, 2) and 3) are equivalent. We now prove that 2) implies 1). Let $\mathcal{V}$ be a variety of exponent $d$ such that $I d(\mathcal{V})=I_{1} \cdots I_{m}$, for some verbally prime T-ideals $I_{1}, \ldots, I_{m}$ and let $\mathcal{U}$ be a proper subvariety of $\mathcal{V}$. By the proof of Theorem 5.1 , there exists a minimal superalgebra $A$ such that $\operatorname{var}(G(A)) \subseteq \mathcal{U}$ and $\exp (G(A))=\exp (\mathcal{U})$. Let $\operatorname{Id}(\mathcal{U})=\operatorname{Id}(G(A))=Q_{1} \cdots Q_{n}$, a product of verbally prime T-ideals. Since $\mathcal{U} \varsubsetneqq \mathcal{V}$ then $I_{1} \cdots I_{m} \varsubsetneqq Q_{1} \cdots Q_{n}$ and by Lemma 7.3 we get that

$$
\exp (\mathcal{U})=\exp \left(Q_{1} \cdots Q_{n}\right)<\exp \left(I_{1} \cdots I_{m}\right)=\exp (\mathcal{V}) .
$$

Thus $\mathcal{V}$ is a minimal variety.

Corollary 7.6. For any integer $d \geq 2$, there exists only a finite number of minimal varieties of exponent $d$. 


\section{Some APplicAtions}

The classification of minimal varieties obtained in the previous sections is an efficient tool for the study of the growth of a variety. To this end we shall give two applications. In the first we shall show an effective way of computing the exponent of a T-ideal given by a set of generators; in the second we shall study the general problem of determining which functions can appear as the growth function of a codimension sequence.

Let $\mathcal{V}$ be a variety of algebras and suppose that $I=I d(\mathcal{V})=\left\langle f_{1}, \ldots, f_{m}\right\rangle_{T}$, i.e., the T-ideal of identities of $\mathcal{V}$ is generated by the polynomials $f_{1}, \ldots, f_{m}$. Then by definition, $\exp (\mathcal{V})$ is the maximal value of $\exp (\mathcal{U})$, where $\mathcal{U}$ varies among all minimal varieties contained in $\mathcal{V}$. In other words,

$$
\exp (\mathcal{V})=\max \{\exp (G(A))\}
$$

where $A$ runs among all minimal superalgebras such that $G(A)$ satisfies the identities $f_{1} \equiv 0, \ldots, f_{m} \equiv 0$.

In order to illustrate this by an example, we consider the identity of Lie solvability: define $s_{1}\left(x_{1}, x_{2}\right)=\left[x_{1}, x_{2}\right]$ and inductively

$$
s_{m+1}\left(x_{1}, \ldots, x_{2^{m+1}}\right)=\left[s_{m}\left(x_{1}, \ldots, x_{2^{m}}\right), s_{m}\left(x_{2^{m}+1}, \ldots, x_{2^{m+1}}\right)\right] .
$$

In short, for any polynomial $f \in F\langle X\rangle$ let us write $\exp (f)=\exp \left(\langle f\rangle_{T}\right)$, the exponent of the T-ideal generated by $f$. We remark that the exponent of several important classes of polynomials has been computed in [5]. We have

Theorem 8.1. For any $m \geq 1, \exp \left(s_{m}\right)=2^{m-1}$.

Proof. For any $m \geq 1$, let $L_{m}=\left\langle s_{m}\right\rangle_{T}$. Then $L_{1}=[F\langle X\rangle, F\langle X\rangle]$ and $L_{m+1}=$ $\left[L_{m}, L_{m}\right]$, for all $m \geq 1$.

If $m=1$, then $c_{n}\left(L_{1}\right)=1$, for all $n$, and this implies that $\exp \left(s_{1}\right)=1=2^{0}$. Let $m \geq 2$ and consider all minimal varieties $\mathcal{V}$ such that $s_{m} \in \operatorname{Id}(\mathcal{V})$, i.e., at the light of Lemma 3.4 (see also the proof of Theorem 5.1), we should consider all possible products $I_{1} \cdots I_{k}$ of verbally prime T-ideals containing $L_{m}$. Note first that if $U T_{2^{m-1}}$ denotes the algebra of $2^{m-1} \times 2^{m-1}$ upper triangular matrices over $F$, then $U T_{2^{m-1}}$ satisfies $s_{m} \equiv 0$. Moreover $U T_{2^{m-1}}$ is a minimal superalgebra with trivial grading and with exponent $2^{m-1}$ (see [11]). Hence

$$
\exp \left(s_{m}\right) \geq \exp \left(U T_{2^{m-1}}\right)=2^{m-1}
$$

Now let $A=A_{s s}+J$ be a minimal superalgebra, where $A_{s s}=A_{1} \oplus \cdots \oplus A_{k}, A_{j}$ are simple superalgebras, and $I d(G(A))=I_{1} \cdots I_{k}$ with $I_{j}=I d\left(G\left(A_{j}\right)\right), j=1, \ldots, k$. Suppose that $I_{1} \ldots I_{k} \supseteq L_{m}$. Then $I_{j} \supseteq L_{m}$, and so $G\left(A_{j}\right)$ satisfies $s_{m} \equiv 0$, for all $j$. Recalling the classification of the simple superalgebras (section [3), a direct inspection shows that either $A_{j} \cong F$ satisfies $s_{1} \equiv 0$, or $A_{j} \cong M_{1,1}(F)$ and $G\left(A_{j}\right)$ satisfies $s_{3} \equiv 0$ but $s_{2} \not \equiv 0$, or $A_{j} \cong F \oplus t_{j} F, t_{j}^{2}=1$, and $G\left(A_{j}\right)$ satisfies $s_{2} \equiv 0$ but $s_{1} \not \equiv 0$.

First let $k \geq 2$. Since $L_{m}=\left[L_{m-1}, L_{m-1}\right] \subseteq I_{1} \cdots I_{k}$, by Theorem 5.3 and Proposition 5.4 we obtain that either $L_{m-1} \subseteq I_{1} \cdots I_{k}$ or there exists $1 \leq r \leq k-1$ such that $L_{m-1} \subseteq\left(I_{1} \cdots I_{r}\right) \cap\left(I_{r+1} \cdots I_{k}\right)$. In the first case, by induction on $m$, $\exp (G(A)) \leq 2^{m-2}$. But $\exp \left(s_{m}\right) \geq 2^{m-1}$ by (8.1); hence $A$ can be excluded. Thus, if $k \geq 2$,

$$
\exp \left(s_{m}\right) \leq \exp \left(I_{1} \cdots I_{r}\right)+\exp \left(I_{r+1} \cdots I_{k}\right) \leq 2^{m-2}+2^{m-2}=2^{m-1}
$$

and this, together with (8.1), gives the desired conclusion. 
If $k=1$ and $m \geq 4$, by the discussion above, $\exp (G)=2<2^{m-1}, \exp \left(M_{1,1}(G)\right)$ $=4<2^{m-1}$. For $m=2$ or 3 one has $\exp \left(s_{2}\right)=\exp (G)=2=2^{1}, \exp \left(s_{3}\right)=$ $\exp \left(M_{1,1}(G)\right)=4=2^{2}$.

We now pass to the second application of the classification of minimal varieties regarding the asymptotic behavior of the codimensions. Namely, we can ask for which functions $f(n)$ of a natural argument there exists a PI-algebra $A$ such that $f(n) \simeq c_{n}(A)$ for some equivalence $\simeq$ of functions. Problems of this type have been considered in various areas.

For instance if GK- $\operatorname{dim}(A)$ denotes the Gelfand-Kirillov dimension of an algebra $A$, it is well known that for any real number $\alpha \geq 2$ there exists an algebra $A$ such that GK- $\operatorname{dim}(A)=\alpha$ whereas the only real numbers $<2$ that can be realized as the GK-dimension of an algebra are 0 and 1 ([22]).

In group theory the so-called distortion functions were considered allowing the embedding of an arbitrary group in a finitely presented group. The problem of describing such functions was posed by Gromov in [17], and in [25] Ol'shanskii showed that any "reasonable" function can occur as a distortion function. In particular $n^{\theta}$, where $\theta \geq 1$ is any "computable" real number, can be realized as the distortion function of the embedding of the infinite cyclic group into a finitely presented group.

Concerning the theory of PI-algebras, as it was mentioned above, the codimension sequence of a non-trivial variety behaves asymptotically as $d^{n}$, for some integer $d$. More precisely, the limit $\lim _{n \rightarrow \infty} \sqrt[n]{c_{n}(\mathcal{V})}=d$ exists and is a non-negative integer (11], [12]). A more precise asymptotic behavior of the codimensions was conjectured by Regev (see for instance 28] for a good survey on this subject). In general, if $f$ and $g$ are two functions of a natural argument, define $f(n) \simeq g(n)$ if

$$
\lim _{n \rightarrow \infty} \frac{f(n)}{g(n)}=1
$$

Regev conjectured that for any non-trivial variety $\mathcal{V}$, there exist constants $C, e, d$ such that

$$
c_{n}(\mathcal{V}) \simeq C n^{e} d^{n} .
$$

Moreover $d$ is an integer and $e \in \frac{1}{2} \mathbb{Z}$.

In this direction we first prove the following result

Theorem 8.2. For any positive integer $d$ there exists a non-zero constant $\alpha$ such that

$$
c_{n}(\mathcal{V}) \geq \alpha n^{\frac{1-d}{2}} d^{n}
$$

for any variety $\mathcal{V}$ with $\exp (\mathcal{V})=d$.

Proof. First recall that the asymptotics for the verbally prime T-ideals were computed by Regev in [27] and Berele and Regev in [3. Namely,

$$
\begin{gathered}
c_{n}\left(M_{k}(F)\right) \simeq \alpha n^{-\frac{k^{2}-1}{2}} k^{2 n}, \\
\alpha_{1} n^{-\frac{k^{2}+l^{2}-1}{2}}(k+l)^{2 n} \leq c_{n}\left(M_{k, l}(G)\right) \leq \alpha_{2} n^{-\frac{k^{2}+l^{2}-1}{2}}(k+l)^{2 n}
\end{gathered}
$$

and

$$
\beta_{1} n^{-\frac{2 k^{2}-1}{2}}\left(2 k^{2}\right)^{n} \leq c_{n}\left(M_{k}(G) \oplus t M_{k}(G)\right) \leq \beta_{2} n^{-\frac{(k-1)^{2}}{2}}\left(2 k^{2}\right)^{n}
$$

for some constants $\alpha, \alpha_{1}, \alpha_{2}, \beta_{1}, \beta_{2}$ such that $\alpha, \alpha_{1}, \beta_{1}>0$. 
Note that as a consequence, we have $\exp \left(M_{k}(F)\right)=k^{2}, \exp \left(M_{k, l}(G)\right)=(k+l)^{2}$, $\exp \left(M_{k}(G)\right)=2 k_{2}$ and,

$$
\frac{c_{n}(\mathcal{V})}{d^{n}} \geq \alpha n^{\frac{1-d}{2}}
$$

for any prime variety $\mathcal{V}$ with $\exp (\mathcal{V})=d$, for some constant $\alpha$.

Now let $A, A_{1}, A_{2}$ be PI-algebras such that $\operatorname{Id}(A)=\operatorname{Id}\left(A_{1}\right) \operatorname{Id}\left(A_{2}\right)$. Then, by 28, Lemma 7.6], if

$$
\alpha_{1, i} n^{v_{i}} d_{i}^{n} \leq c_{n}\left(A_{i}\right) \leq \alpha_{2, i} n^{u_{i}} d_{i}^{n}
$$

for some $\alpha_{1, i}, \alpha_{2, i}>0, v_{i} \leq u_{i}, i=1,2$, then

$$
\beta_{1} n^{v_{1}+v_{2}+1}\left(d_{1}+d_{2}\right)^{n} \leq c_{n}(A) \leq \beta_{2} n^{u_{1}+u_{2}+1}\left(d_{1}+d_{2}\right)^{n}
$$

for some constants $\beta_{1}, \beta_{2}>0$. Now let $\mathcal{V}$ be any minimal variety with $\operatorname{Id}(\mathcal{V})=$ $I_{1} \cdots I_{k}$ where $I_{1}, \ldots, I_{k}$ are verbally prime T-ideals. Then, combining (8.2) and $(8.3)$, we obtain

$$
\frac{c_{n}(\mathcal{V})}{d^{n}} \geq \alpha n^{\left(\frac{1-d_{1}}{2}+\cdots+\frac{1-d_{k}}{2}\right)+k-1}=\alpha n^{\frac{k-\left(d_{1}+\cdots+d_{k}\right)}{2}+k-1} \geq \alpha n^{\frac{1-d}{2}}
$$

where for $j=1, \ldots, k d_{j}=\exp \left(I_{j}\right)$ and $d=d_{1}+\cdots+d_{k}=\exp (\mathcal{V})$. This proves the theorem.

The inverse problem of what constants $C$ and half integers $e$ can be realized among codimension growth functions was discussed in [10] and [18]. The precise asymptotics have been computed for only very few varieties, and in all cases the conjecture was confirmed.

In 14 it was shown that for any $d \geq 2$ there exists a variety $\mathcal{V}$ with $\exp (\mathcal{V})=d$.

For the second approximation we need to compute the polynomial factor $n^{e}$. In this case the inverse problem can be formulated as follows: describe a subset $E$ of the real numbers such that for any $e \in E$ there exists a PI-algebra $A$ with $c_{n}(A) \simeq C n^{e} d^{n}$. More precisely,

$$
e=\lim _{n \rightarrow \infty} \log _{n} \frac{c_{n}(A)}{d^{n}}
$$

exists and $e \in E$ where $d=\exp (A)$. According to Regev's conjecture, $E \subseteq \frac{1}{2} \mathbb{Z}$. In order to construct varieties with the same exponent $d$ but with distinct polynomial factors, we shall use the following result of Guterman and Regev

Lemma 8.3 ([18, Corollary 2.6]). Let $R$ be a PI-algebra over $F$, char $F=0$. Suppose that for any $r \in R, r \neq 0$, there exist $r^{\prime}, r^{\prime \prime} \in R$ such that $r r^{\prime} \neq 0$ and $r^{\prime \prime} r \neq 0$. If $k \geq 2$ and $A \subseteq M_{k}(R)$ is the subalgebra defined by

$$
A=R e_{11}+\sum_{1 \leq i<j \leq k} R e_{i j}
$$

then $c_{n}(A)=n(n-1) \cdots(n-k+2) c_{n-k+1}(R)$.

In the following theorem we use the well-known Lagrange theorem stating that any positive integer can be written as the sum of at most four squares. In order to simplify the notation we write $d=k_{1}^{2}+\cdots+k_{j}^{2}$ if $d$ is the sum of $j$ squares, but it cannot be written as the sum of $i<j$ squares. 
Theorem 8.4. Given any integer $d \geq 2$, there exists a constant $0 \leq \gamma \leq \frac{9}{2}$ such that, for any integer $r \geq 0$, we can find a variety $\mathcal{V}$ with the property that

$$
c_{n}(\mathcal{V}) \simeq \beta n^{e} d^{n}
$$

where $\beta$ is a constant and $e=\frac{1-d}{2}+\gamma+r$. Moreover, $\gamma=0, \frac{3}{2}, 3, \frac{9}{2}$ according as $d=k^{2}, k_{1}^{2}+k_{2}^{2}, k_{1}^{2}+k_{2}^{2}+k_{3}^{2}, k_{1}^{2}+k_{2}^{2}+k_{3}^{2}+k_{4}^{2}$, respectively.

Proof. Let $R=U T\left(d_{1}, \ldots, d_{m}\right)$ be the upper block triangular matrix algebra

$$
U T\left(d_{1}, \ldots, d_{m}\right)=\left(\begin{array}{cccc}
M_{d_{1}}(F) & & * \\
0 & \ddots & \\
\vdots & & & \\
0 & \cdots & 0 & M_{d_{m}}(F)
\end{array}\right) .
$$

Then by [14] (see [13]) $R$ generates a minimal variety and $c_{n}(R) \simeq \alpha n^{g} d^{n}$, where $\alpha$ is a constant, $d=d_{1}^{2}+\cdots+d_{m}^{2}$ and $g=-\frac{1}{2}(d-3 m+2)=\frac{1-d}{2}+\frac{3 m-3}{2}$.

Now, according to whether $d$ can be written as a sum of $m=1$ or 2 or 3 , or 4 squares, we obtain that $g=\frac{1+d}{2}+\gamma$, where $\gamma=0$ or $\frac{3}{2}$ or 3 or $\frac{9}{2}$, respectively. If we now apply to $R$ the procedure of Lemma 8.3 , we obtain an algebra $A$ such that $c_{n}(A) \simeq \beta n^{e} d^{n}$ with $e=\frac{1-d}{2}+\gamma+r$ for any $r=1,2, \ldots$.

\section{REFERENCES}

1. S. A. Amitsur and A. Regev, P.I. algebras and their cocharacters. J. Algebra 78 (1982), 248-254. MR 84b:16020

2. A. Berele, Cocharacters of Z/2Z-graded algebras. Israel J. Math. 61 (1988), 225-234. MR 89h:16018

3. A. Berele and A. Regev, On the codimensions of the verbally prime P.I. algebras. Israel J. Math. 91 (1995), 239-247. MR 96g:16028

4. A. Berele and A. Regev, Codimensions of products and intersections of verbally prime Tideals. Israel J. Math. 103 (1998), 17-28. MR 99b:16037

5. A. Berele and A. Regev, Exponential growth for codimensions of some p.i. algebras. J. Algebra 241 (2001), 118-145. MR 2002k:16046

6. G. M. Bergman and J. Lewin, The semigroup of ideals of a fir is (usually) free. J. London Math. Soc. (2) 11 (1975), 21-31. MR 52:459

7. V. Drensky, Extremal varieties of algebras I. (Russian) Serdica 13 (1987), 320-332. MR 90e:08010

8. V. Drensky, Extremal varieties of algebras II. (Russian) Serdica 14 (1988), 20-27. MR 90e:08011

9. V. Drensky, Gelfand-Kirillov dimension of PI-algebras, In: Methods in Ring Theory, Lect. Notes in Pure and Appl. Math., Vol. 198 (1998), 97-113. MR 2001g:16042

10. V. Drensky and A. Regev, Exact asymptotic behaviour of the codimensions of some P.I. algebras. Israel J. Math. 96 (1996), 231-242. MR 98b:16021

11. A. Giambruno and M. Zaicev, On codimension growth of finitely generated associative algebras. Adv. Math. 140 (1998), 145-155. MR 99k:16049

12. A. Giambruno and M. Zaicev, Exponential codimension growth of P.I. algebras: an exact estimate. Adv. Math. 142 (1999), 221-243. MR 2000a:16048

13. A. Giambruno and M. Zaicev, Minimal varieties of algebras of exponential growth, Electron. Res. Announc. Amer. Math. Soc. 6 (2000), 40-44. MR 2001e:16037

14. A. Giambruno and M. Zaicev, Minimal varieties of exponential growth. Adv. Math. 174 (2003), 310-323.

15. A. Giambruno and M.V. Zaicev, Minimal varieties of given codimension growth. Vestnik Moskov. Univ. Ser I Mat. Mekh. (2003), No. 1, 20-22.

16. A. Giambruno and M. Zaicev, A characterization of varieties of associative algebras of exponent two. Serdica Math.J. 26 (2000), 245-252. MR 2001i:16041 
17. M.L. Gromov, Geometric group theory, vol. 2 (Sussex, 1991), Cambridge Univ. Press Cambridge, 1993. MR 95m:20041

18. A. Guterman and A. Regev, On the growth of identities, In: Algebra (Moscow, 1998), 319-330, de Gruyter, Berlin, 2000. MR 2001a:16039

19. G. James and A. Kerber, The representation theory of the symmetric group, Encyclopedia of Mathematics and its Applications, vol. 16, Addison-Wesley, London, 1981. MR 83k:20003

20. A. Kemer, T-ideals with power growth of the codimensions are Specht. (Russian) Sibirskii Matematicheskii Zhurnal 19 (1978), 54-69; English translation: Siberian Math. J. 19 (1978), 37-48. MR 57:6070

21. A. Kemer, Ideals of identities of associative algebras, AMS Translations of Mathematical Monograph, vol. 87, 1988. MR 92f:16031

22. G. R. Krause and T. H. Lenagan, Growth of algebras and Gelfand-Kirillov dimension, Revised edition, Graduate Texts in Mathematics vol. 22, Amer. Math. Soc. Providence, R.I., 2000. MR 2000j: 16035

23. V. N. Latyshev, On Regev's theorem on identities in tensor product of PI-algebras. Ups. Mat. Nauk. 27 (1973), 213-214 (Russian). MR 52:13924

24. J. Lewin, A matrix representation for associative algebras I. Trans. Amer. Math. Soc. 188 (1974), 293-308. MR 49:2848

25. A. Yu. Ol'shanskii, On the distorsion of subgroups of finitely presented groups. (Russian) Mat. Sb. 188 (1997), 51-98; translation in Sb. Math. 188 (1997), 1617-1664. MR 99a:20038

26. A. Regev, Existence of identities in $A \otimes B$. Israel J. Math. 11 (1972), 131-152. MR 47:3442

27. A. Regev, Codimensions and trace codimensions of matrices are asymptotically equal. Israel J. Math. 47 (1984), 246-250. MR 85j:16024

28. A. Regev, Asymptotics of codimensions of some P.I. algebras In: Trends in Ring Theory, CMS Conference Proc. Vol. 22, Amer. Math. Soc., Providence, R.I., 1998, 159-172. MR 98k:16033

29. L. H. Rowen, Polynomial Identities in Ring Theory, Academic Press, New York, 1980. MR 82a:16021

30. A. N. Stoyanova-Venkova, Some lattices of varieties of associative algebras defined by identities of fifth degree. (Russian), C. R. Acad. Bulg. Sci. 35 (1982), 865-868. MR 84b:08007

31. E. J. Taft, Invariant Wedderburn factors. Illinois J. Math. 1 (1957), 565-573. MR 20:4586

Dipartimento di Matematica ed Applicazioni, Universitì di Palermo, Via Archirafi 34, 90123 PALermo, Italy

E-mail address: agiambr@unipa.it

Department of Algebra, Faculty of Mathematics and Mechanics, Moscow State UniVERsity, Moscow, 119992 Russia

E-mail address: zaicev@mech.math.msu.su 